\title{
SPECTRAL APPROXIMATION OF BANDED LAURENT MATRICES WITH LOCALIZED RANDOM PERTURBATIONS
}

\author{
A. Böttcher, M. Embree, M. Lindner
}

This paper explores the relationship between the spectra of perturbed infinite banded Laurent matrices $L(a)+K$ and their approximations by perturbed circulant matrices $C_{n}(a)+P_{n} K P_{n}$ for large $n$. The entries $K_{j k}$ of the perturbation matrices assume values in prescribed sets $\Omega_{j k}$ at the sites $(j, k)$ of a fixed finite set $E$, and are zero at the sites $(j, k)$ outside $E$. With $\mathcal{K}_{\Omega}^{E}$ denoting the ensemble of these perturbation matrices, it is shown that

$$
\lim _{n \rightarrow \infty} \bigcup_{K \in \mathcal{K}_{\Omega}^{E}} \operatorname{sp}\left(C_{n}(a)+P_{n} K P_{n}\right)=\bigcup_{K \in \mathcal{K}_{\Omega}^{E}} \operatorname{sp}(L(a)+K)
$$

under several fairly general assumptions on $E$ and $\Omega$.

\section{Introduction and main results}

Given a continuous complex-valued function $a$ on the complex unit circle $\mathbf{T}$, we denote by $\left\{a_{n}\right\}_{n \in \mathbf{Z}}$ the sequence of its Fourier coefficients,

$$
a_{n}=\frac{1}{2 \pi} \int_{0}^{2 \pi} a\left(e^{i \theta}\right) e^{-i n \theta} d \theta
$$

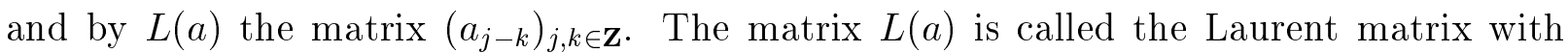
the symbol $a$, and it is well known that $L(a)$ induces a bounded operator on $\ell^{2}(\mathbf{Z})$ whose spectrum is the range $a(\mathbf{T})$ of $a, \operatorname{sp} L(a)=a(\mathbf{T})$.

Let $\mathcal{P}$ denote the set of all trigonometric polynomials. Thus, $a \in \mathcal{P}$ if and only if $a_{n} \neq 0$ for at most finitely many $n$. Throughout what follows we assume that $a \in \mathcal{P}$, which is equivalent to the requirement that $L(a)$ be a banded matrix.

For $a \in \mathcal{P}$, we define the $n \times n$ matrix $C_{n}(a)$ by $C_{n}(a)=\left(c_{j k}\right)_{j, k=1}^{n}$ with $c_{j k}=a_{j-k(\bmod n)}$. The matrix $C_{n}(a)$ is a circulant matrix provided $n$ exceeds the bandwidth of $L(a)$. For 
example, if $a(t)=a_{-1} t^{-1}+a_{0}+a_{1} t+a_{2} t^{2}(t \in \mathbf{T})$, then

$$
C_{6}(a)=\left(\begin{array}{cccccc}
a_{0} & a_{-1} & 0 & 0 & a_{2} & a_{1} \\
a_{1} & a_{0} & a_{-1} & 0 & 0 & a_{2} \\
a_{2} & a_{1} & a_{0} & a_{-1} & 0 & 0 \\
0 & a_{2} & a_{1} & a_{0} & a_{-1} & 0 \\
0 & 0 & a_{2} & a_{1} & a_{0} & a_{-1} \\
a_{-1} & 0 & 0 & a_{2} & a_{1} & a_{0}
\end{array}\right) .
$$

The spectrum of $C_{n}(a)$ is $a\left(\mathbf{T}_{n}\right)$, where $\mathbf{T}_{n}$ denotes the set of the $n$th unit roots. Thus, the spectrum of $C_{n}(a)$ approximates the spectrum of $L(a)$ as $n \rightarrow \infty$.

This paper addresses the relationship between the spectra of $L(a)+K$ and $C_{n}(a)+$ $P_{n} K P_{n}$ as $n \rightarrow \infty$ when $K=\left(K_{j k}\right)$ is a (deterministic or random) matrix whose nonzero entries are all situated in a fixed finite set $E$ of sites $(j, k)$ with $j, k \in\{1, \ldots, m\}$, and where $P_{n} K P_{n}=\left(K_{j k}\right)_{j, k=1}^{n}$. Related problems arise in the discretization of initial-boundary value problems [1], [8], [14], the theory of linear systems with uncertain data [7], [12], [13], smallworld networks [20], population biology [16], and non-Hermitian quantum mechanics [4], [5], [6], [10], [21]. A prominent question is whether the spectrum of $L(a)+K$ can be found by replacing $L(a)+K$ with $C_{n}(a)+P_{n} K P_{n}$, where $n$ is large, and then computing the eigenvalues of $C_{n}(a)+P_{n} K P_{n}$ numerically. The reverse question is also of interest: Sometimes the spectrum of $L(a)+K$ is known, and the problem is whether this tells us anything about the eigenvalues of $C_{n}(a)+P_{n} K P_{n}$ for large $n$. In the language of physics, we are here concerned with the problem of whether the passage from the "finite volume case" to the "infinite volume case" is continuous or not.

One can show that $\operatorname{sp} L(a)=a(\mathbf{T})$ is always a subset of $\operatorname{sp}(L(a)+K)$. Thus

$$
\operatorname{sp}(L(a)+K)=\operatorname{sp} L(a) \cup X=a(\mathbf{T}) \cup X
$$

with some (possibly empty) set $X$, all points of which are eigenvalues of $L(a)+K$. The difficulty with spectral approximation is that $X$ may contain entire connected components of $\mathbf{C} \backslash a(\mathbf{T})$, which, moreover, can emerge suddenly even if $K$ changes continuously. For instance, if $a(t)=t(t \in \mathbf{T})$, in which case $L(a)$ is the forward shift on $\ell^{2}(\mathbf{Z})$, and $K$ is the matrix whose $(2,1)$ entry is $\omega$ with all other entries zero, then

$$
\operatorname{sp}(L(a)+K)= \begin{cases}\mathbf{T} & \text { if } \omega \neq-1 \\ \overline{\mathbf{D}} & \text { if } \omega=-1\end{cases}
$$

where $\overline{\mathbf{D}}=\{\lambda \in \mathbf{C}:|\lambda| \leq 1\}$, while, for $n \geq 2$,

$$
\operatorname{sp}\left(C_{n}(a)+P_{n} K P_{n}\right)=\left\{\lambda \in \mathbf{C}: \lambda^{n}=1+\omega\right\},
$$

which shows that $\operatorname{sp}\left(C_{n}(a)+P_{n} K P_{n}\right)$ does not approximate $\operatorname{sp}(L(a)+K)$ if $w=-1$. (See Example 4.1 for further details.)

Given a nonempty compact set $M \subset \mathbf{C}$ and a sequence $\left\{M_{n}\right\}$ of nonempty compact sets $M_{n} \subset \mathbf{C}$, we write $\lim M_{n}=M$ if $M_{n}$ converges to $M$ in the Hausdorff metric. The closure of a set $G \subset \mathbf{C}$ will be denoted by $\bar{G}$ or clos $G$, and its boundary $\bar{G} \backslash G$ by $\partial G$. 
Theorem 1.1 If a connected component $G$ of $\mathbf{C} \backslash a(\mathbf{T})$ is not entirely contained in the set $\operatorname{sp}(L(a)+K)$, then

$$
\lim _{n \rightarrow \infty}\left(\left(\operatorname{sp}\left(C_{n}(a)+P_{n} K P_{n}\right) \cap G\right) \cup \partial G\right)=\operatorname{sp}(L(a)+K) \cap \bar{G} .
$$

Equality (1) holds in particular if $G$ is the unbounded connected component of $\mathbf{C} \backslash a(\mathbf{T})$.

We will show that (1) is in general no longer true if $G$ is contained in $\operatorname{sp}(L(a)+K)$.

Here is a result for perturbations localized on the main diagonal or in a single site. For $a \in \mathcal{P}$, let $B(a)$ be the union of $a(\mathbf{T})$ and all bounded components of $\mathbf{C} \backslash a(\mathbf{T})$. Furthermore, for $\rho>0$, define $a_{\rho} \in \mathcal{P}$ by $a_{\rho}(t)=a(\rho t)(t \in \mathbf{T})$. Thus, if $a(t)=\sum_{k} a_{k} t^{k}$, then $a_{\rho}(t)=\sum_{k} a_{k} \rho^{k} t^{k}$. Finally, put

$$
B^{*}(a)=\bigcap_{\rho>0} B\left(a_{\rho}\right)
$$

Theorem 1.2 Let $G$ be a connected component of $\mathbf{C} \backslash a(\mathbf{T})$.

(a) Finitely-Many Main Diagonal Perturbations. If $K=\operatorname{diag}\left(K_{11}, \ldots, K_{m m}\right)$ and $G$ contains at least one point of $\mathbf{C} \backslash B^{*}(a)$, then equality (1) is true.

(b) Single-Entry Perturbations. If $K$ is a matrix whose $(j, k)$ entry is $\omega$ with all other entries zero, and if the $(k-j)$ th Fourier coefficient of $1 /(a-\lambda)$ is either identically zero in $G$ or nowhere locally constant in $G$, then equality (1) is valid.

For tridiagonal Laurent matrices with a finite number of perturbed diagonal entries, we have the following.

Corollary 1.3 If $a(t)=t+\alpha^{2} t^{-1}(t \in \mathbf{T})$ with $\alpha \in[0,1]$ and $K=\operatorname{diag}\left(K_{11}, \ldots, K_{m m}\right)$, then

$$
\lim _{n \rightarrow \infty}\left(\operatorname{sp}\left(C_{n}(a)+P_{n} K P_{n}\right) \cup a(\mathbf{T})\right)=\operatorname{sp}(L(a)+K) .
$$

These three results are related to the results of [1], [8], [14] and are perhaps known to specialists. We state and prove these results here for the reader's convenience and because we have not found them explicitly in the literature.

We now consider the case where $K$ is randomly chosen. Let $E$ be a finite set of sites $(j, k)$ with $j, k \in\{1, \ldots, m\}$ and suppose for each $(j, k) \in E$ we are given a compact subset $\Omega_{j k}$ of the plane that contains the origin. We put $\Omega=\left\{\Omega_{j k}\right\}_{(j, k) \in E}$ and denote by $\mathcal{K}_{\Omega}^{E}$ the set of all matrices $K=\left(K_{j k}\right)$ for which $K_{j k} \in \Omega_{j k}$ if $(j, k) \in E$ and $K_{j k}=0$ if $(j, k) \notin E$. Finally, for a finite or infinite matrix $A$, we define

$$
\operatorname{sp}_{\Omega}^{E} A=\bigcup_{K \in \mathcal{K}_{\Omega}^{E}} \operatorname{sp}(A+K) .
$$

Clearly, we may think of $\operatorname{sp}_{\Omega}^{E} A$ as the union of all possible spectra that may emerge when perturbing $A$ by a matrix $K$ randomly chosen in $\mathcal{K}_{\Omega}^{E}$. 
Each of the previous three results has a direct analogue relating $\operatorname{sp}_{\Omega}^{E} C_{n}(a)$ to $\operatorname{sp}_{\Omega}^{E} L(a)$. These results do not use any specific knowledge of the probability distributions specifying how perturbations are drawn from $\Omega$, but only depend upon the support of the perturbations; see Davies [4] for a similar approach. Knowledge of distributions may lead to more precise statements about the rate at which spectral limits are reached; see [21].

Theorem 1.4 Let $G$ be a connected component of $\mathbf{C} \backslash a(\mathbf{T})$ and suppose $G$ is not contained in $\operatorname{sp}_{\Omega}^{E} L(a)$. Then

$$
\lim _{n \rightarrow \infty}\left(\operatorname{sp}_{\Omega}^{E} C_{n}(a) \cap \bar{G}\right)=\operatorname{sp}_{\Omega}^{E} L(a) \cap \bar{G} .
$$

In particular, (4) is true if $G$ is the unbounded connected component of $\mathbf{C} \backslash a(\mathbf{T})$.

Theorem 1.5 Let $G$ be a connected component of $\mathbf{C} \backslash a(\mathbf{T})$.

(a) Finitely-Many Main Diagonal Perturbations. If $E=\{(1,1), \ldots,(m, m)\}$ and $G$ contains at least one point of $\mathbf{C} \backslash B^{*}(a)$, then equality (4) holds.

(b) Single-Entry Perturbations. If $E=\{(j, k)\}$ and if the $(k-j)$ th Fourier coefficient of $1 /(a-\lambda)$ is either identically zero in $G$ or nowhere locally constant in $G$, then equality (4) is satisfied.

Corollary 1.6 Let $E=\{(1,1), \ldots,(m, m)\}$. If $a(t)=t+\alpha^{2} t^{-1}(t \in \mathbf{T})$ with $\alpha \in[0,1]$, then

$$
\lim _{n \rightarrow \infty} \operatorname{sp}_{\Omega}^{E} C_{n}(a)=\operatorname{sp}_{\Omega}^{E} L(a)
$$

A set $S \subset \mathbf{C}$ is said to be starlike if it, together with each of its points $\omega$, contains the line segment $[0, \omega]$. We let $S^{\circ}$ denote the set of all interior points of $S$. For $\varepsilon>0$, we define $\varepsilon S$ as $\{\varepsilon \omega: \omega \in S\}$ and $\varepsilon \Omega$ as $\left\{\varepsilon \Omega_{j k}\right\}_{(j, k) \in E}$.

Theorem 1.7 If each $\Omega_{j k}$ is a starlike compact nonempty set such that $\Omega_{j k}=$ clos $\Omega_{j k}^{\circ}$, then

$$
\lim _{n \rightarrow \infty} \operatorname{sp}_{\varepsilon \Omega}^{E} C_{n}(a)=\operatorname{sp}_{\varepsilon \Omega}^{E} L(a)
$$

for all $\varepsilon \in(0, \infty)$ except for at most finitely many $\varepsilon_{1}, \ldots, \varepsilon_{\ell}$, where $\ell$ does not exceed the number of bounded components of $\mathbf{C} \backslash a(\mathbf{T})$.

We conjecture that, under the above hypotheses for $\Omega$, equality (5) is actually true for all $\varepsilon \in(0, \infty)$, but we have not been able to prove this.

For tridiagonal Laurent matrices, we can supplement Theorems 1.4 and 1.7 by the following result, which concerns perturbations in a single site.

Theorem 1.8 Let $E=\{(j, k)\}$ and let $\Omega_{j k}$ be a compact subset of $\mathbf{C}$ containing the origin. Furthermore, suppose $a(t)=t+\alpha^{2} t^{-1}(t \in \mathbf{T})$ with $\alpha \in[0,1]$. If $\alpha=1$ or $j-k \neq 1$ or $-1 / \varepsilon \notin \Omega_{j k}$, then (5) is valid. Otherwise, if $\alpha \in[0,1), j-k=1$, and $-1 / \varepsilon \in \Omega_{j k}$, then (5) is not true for $\Omega_{j k}=\{-1 / \varepsilon, 0\}$, while (5) holds if $\Omega_{j k}$ contains, in addition to $-1 / \varepsilon$ and 0 , a third point $\omega_{0}$ and a continuous curve between $-1 / \varepsilon$ and $\omega_{0}$. 
Corollary 1.6 and Theorem 1.8 show in particular that $(5)$ is valid for all $\varepsilon \in(0, \infty)$ if $L(a)$ is tridiagonal, $\Omega$ is a closed ellipse (including the interesting extreme cases of a line segment and of a closed disk) that contains the origin, and $E$ is a singleton or a finite subset of the main diagonal.

The paper is organized as follows. We have produced several pictures in order to demonstrate how spectral approximation works in practice; Section 2 contains the comments to these pictures. In Section 3 we record some well-known results that are used in the subsequent proofs. The proofs of Theorems 1.1 and 1.2 and of Corollary 1.3 are contained in Section 4, while Theorems 1.4, 1.5, 1.7, 1.8 and Corollary 1.6 are proved in Section 5. In Section 6 we briefly address the different limiting behavior observed for Toeplitz and circulant matrices, and conclude in Section 7 with a few conjectures that might stimulate further research.

\section{Illustrations}

We will refer to

$$
\begin{aligned}
a(t)= & (1.5-1.2 i) z^{-1} \\
& +(0.34+0.84 i) z+(-0.46-0.1 i) z^{2}+(0.17-1.17 i) z^{3}+(-1+0.77 i) z^{4}
\end{aligned}
$$

as the animal symbol or the \&ymbol; spectral approximation for this symbol leads to pictures of several animals; Figure 3 shows two birds, while the right column of Figure 10 is reminiscent of horses in cave paintings. The range $a(\mathbf{T})$ and the set $B^{*}(a)$ are shown in Figure 1. Figure 2 provides an indication of the set

$$
\bigcup_{\substack{1 \leq j \leq 5 \\ 1 \leq k \leq 5}} \operatorname{sp}_{[-7,7]}^{(j, k)} L(a),
$$

where $\mathrm{sp}^{(j, k)}$ is an abbreviation for $\mathrm{sp}^{\{(j, k)\}}$. We see that $(6)$ is the union of $a(\mathbf{T})$ and many antennae (which, in the case at hand, replace the "wings" of [3], [10]). Theorem 1.5(b) would guarantee that Figure 2 indeed approximates (6) if we knew that the analytic functions $[1 /(a-\lambda)]_{k-j}$ were nowhere locally constant for $-5 \leq k-j \leq 5$. This is definitely true in the unbounded component of $\mathbf{C} \backslash a(\mathbf{T})$. We have checked numerically that each component of $\mathbf{C} \backslash a(\mathbf{T})$ contains two points $\lambda_{1}$ and $\lambda_{2}$ such that $\left[1 /\left(a-\lambda_{1}\right)\right]_{k-j}$ and $\left[1 /\left(a-\lambda_{2}\right)\right]_{k-j}$ are different. For example, the points $\lambda_{1}=1.5$ and $\lambda_{2}=2.25+0.5 i$ belong to the upper-right component of $\mathbf{C} \backslash a(\mathbf{T})$ (constituting the head and the upper part of the body of the animal \&), and the values of $\left[1 /\left(a-\lambda_{1}\right)\right]_{k-j}$ and $\left[1 /\left(a-\lambda_{2}\right)\right]_{k-j}$ are given in Table 1 . Consequently, we may use Theorem 1.5(b) to conclude that Figure 2 should be a good approximation of the set (6).

Figure 3 shows approximations to $\operatorname{sp}_{[-7,7]}^{E} L(a)$ for the $\&$ symbol when $E=\{(1,1),(2,1)\}$ (top) and $E=\{(1,1),(1,2)\}$ (bottom). By Theorem 1.4, the plots should approximate $\operatorname{sp}_{[-7,7]}^{E} L(a)$ well in the unbounded component of $\mathbf{C} \backslash a(\mathbf{T})$, that is, the birds' bills and tails are close to the truth. Our results are not applicable to the bounded components. 


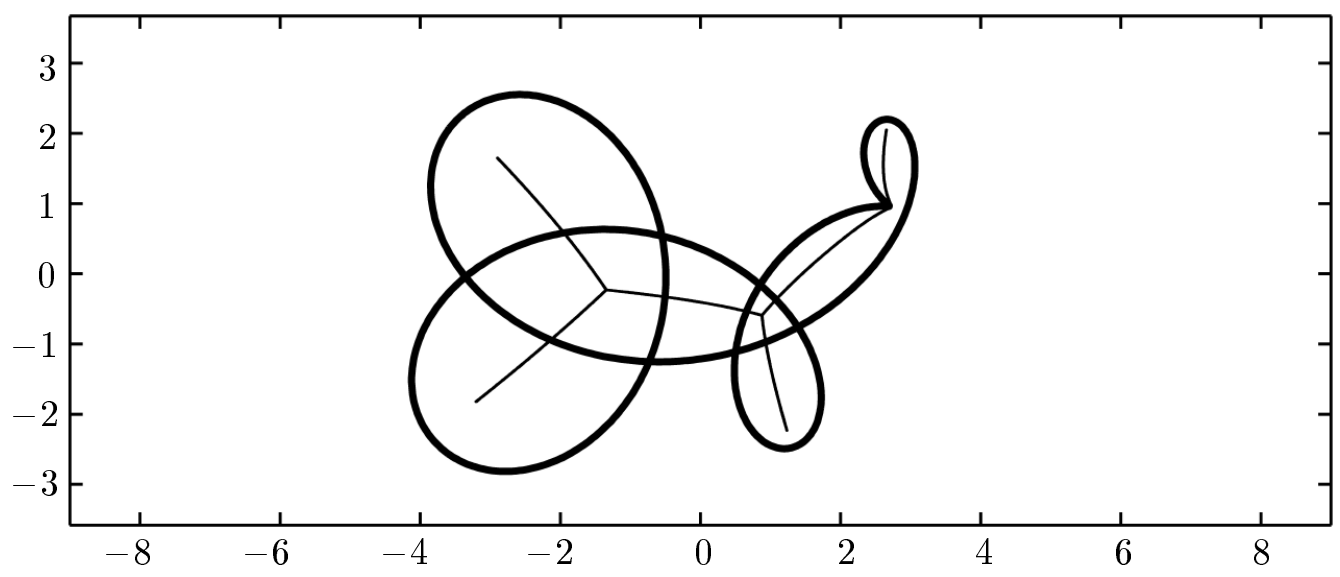

Figure 1: $a(\mathbf{T})$ (thick line) and $B^{*}(a)$ (thin line) for the animal symbol.

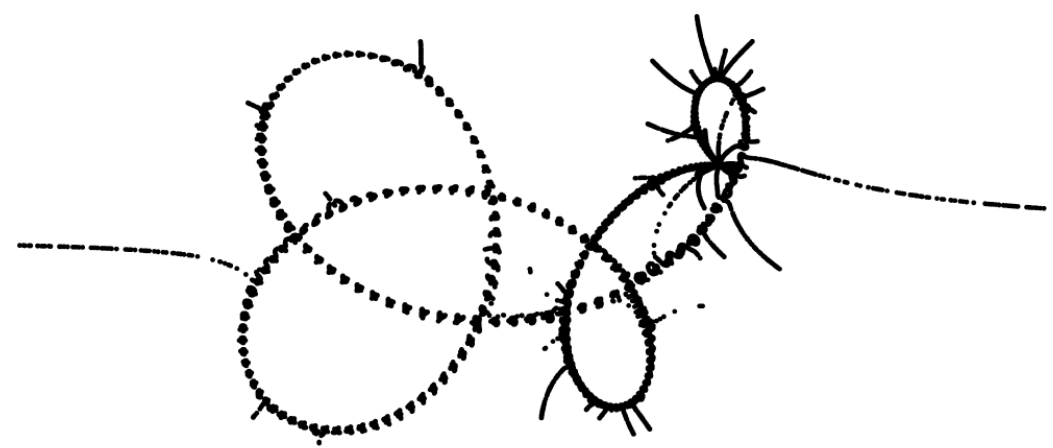

Figure 2: Eigenvalues of $C_{250}(a)$ for the animal symbol, perturbed in one randomly chosen entry in the upper left $5 \times 5$ block by a random number uniformly distributed in $[-7,7]$. This plot superimposes the eigenvalues of 2000 samples.

\begin{tabular}{c|c|c|c|c|} 
& $k-j=-5$ & $k-j=-4$ & $k-j=-3$ & $k-j=-2$ \\
\hline$\lambda_{1}$ & $-0.2037+0.1114 i$ & $-0.1443-0.0654 i$ & $-0.0841+0.0648 i$ & $-0.2849-0.0149 i$ \\
$\lambda_{2}$ & $-0.1500+0.1924 i$ & $-0.0908+0.0331 i$ & $-0.1066+0.1782 i$ & $-0.3709+0.0319 i$
\end{tabular}

\begin{tabular}{c|c|c|c|c|} 
& $k-j=-1$ & $k-j=0$ & $k-j=1$ & $k-j=2$ \\
\hline$\lambda_{1}$ & $-0.1288-0.2019 i$ & $-0.0933-0.0258 i$ & $0.1289+0.1292 i$ & $0.0881+0.0532 i$ \\
$\lambda_{2}$ & $-0.2111-0.2624 i$ & $-0.0734-0.1440 i$ & $0.1884+0.0383 i$ & $0.1294+0.0836 i$
\end{tabular}

\begin{tabular}{c|c|r|r|} 
& $k-j=3$ & \multicolumn{1}{|c}{$k-j=4$} & \multicolumn{1}{c}{$k-j=5$} \\
\hline$\lambda_{1}$ & $0.0499+0.0363 i$ & $0.0146-0.1036 i$ & $-0.0872-0.0707 i$ \\
$\lambda_{2}$ & $0.0391+0.1132 i$ & $-0.0413-0.0248 i$ & $-0.0947-0.0557 i$
\end{tabular}

Table 1: Fourier coefficients of $\left[1 /\left(a-\lambda_{1}\right)\right]_{k-j}$ and $\left[1 /\left(a-\lambda_{2}\right)\right]_{k-j}$ for the animal symbol, where $\lambda_{1}=1.5$ and $\lambda_{2}=2.25+0.5 i$. These coefficients were computed using MATLAB's built-in quadrature routine quadl, and are believed to be correct to the digits presented. 

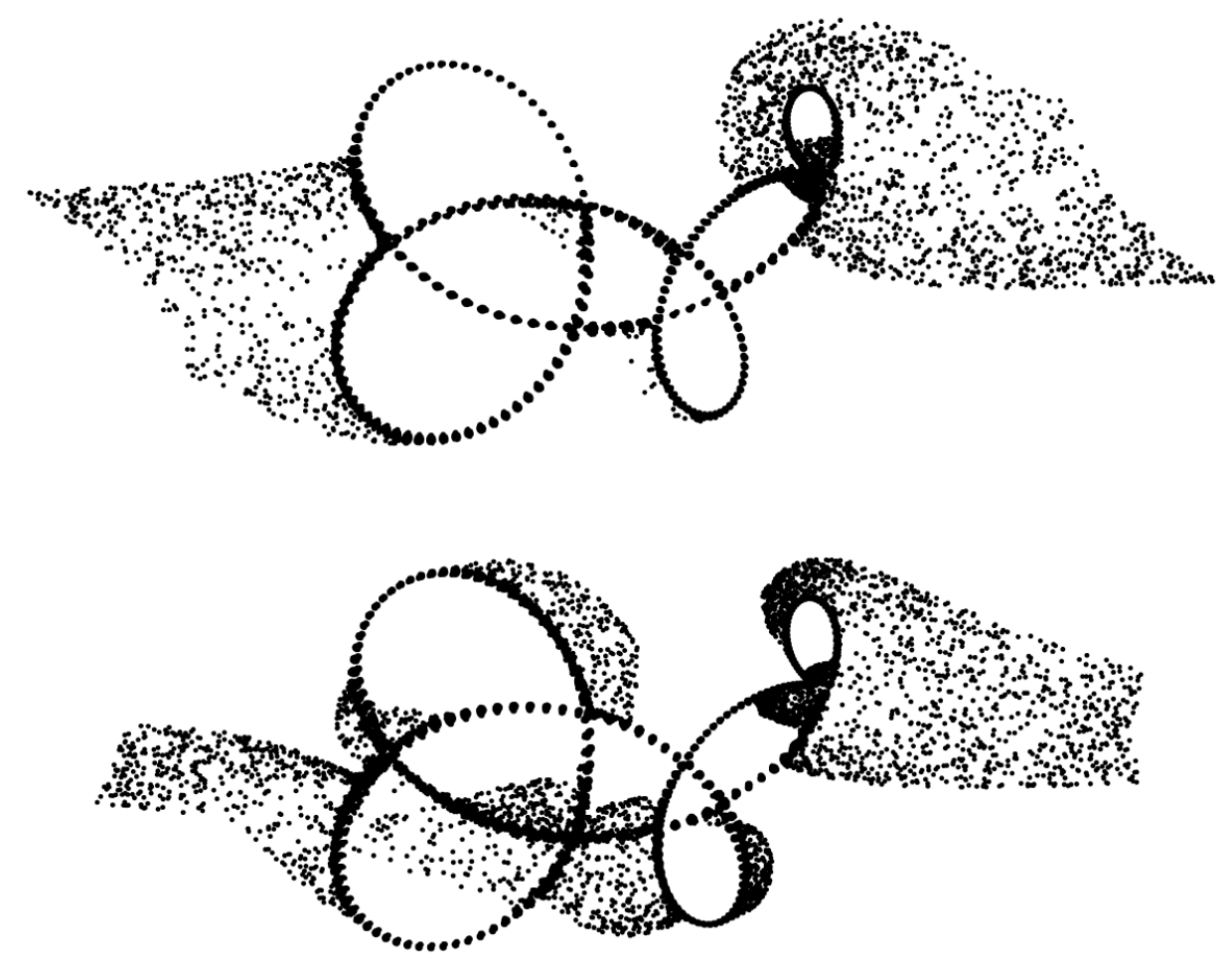

Figure 3: Eigenvalues of $C_{250}(a)$ for the animal symbol, perturbed simultaneously in two entries by random numbers uniformly distributed in $[-7,7]$. On the top, the $(1,1)$ and $(2,1)$ entries are varied; on the bottom, the $(1,1)$ and $(1,2)$ entries are perturbed. Each plot superimposes the eigenvalues of 2000 samples.
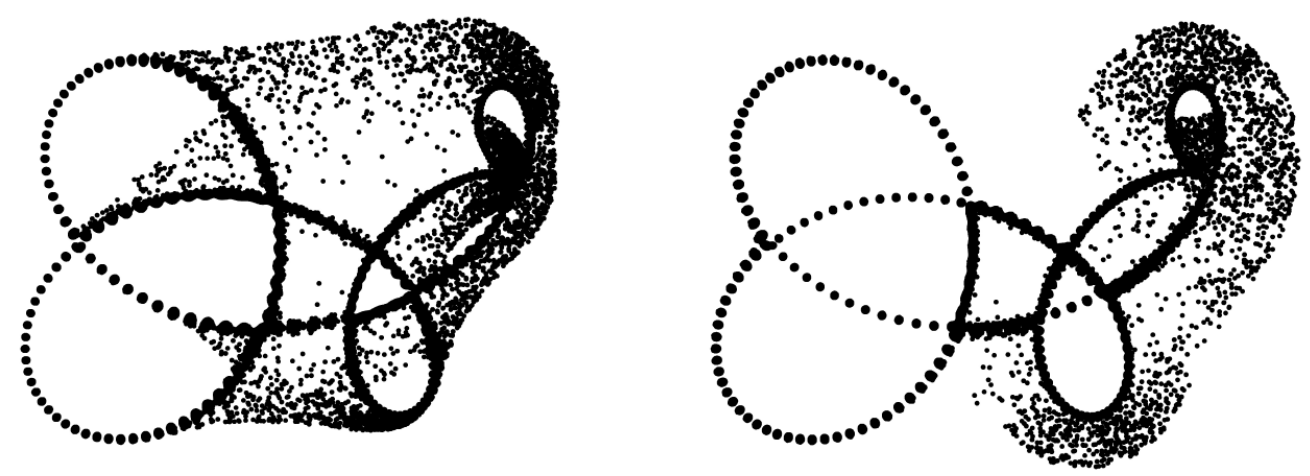

Figure 4: Eigenvalues of $C_{250}(a)$ for the animal symbol, perturbed in the $(j, k)$ entry by a random number uniformly distributed in $7 \overline{\mathbf{D}}$. On the left, we take $(j-k)(\bmod n)=-1$; on the right, $(j-k)(\bmod n)=1$. Each plot superimposes the eigenvalues of 2000 samples. 
In Figure 4 we see approximations to $\operatorname{sp}_{7 \overline{\mathbf{D}}}^{(j, k)} L(a)$ for the $8 y m b o l$ when $(j, k)=(1,2)$ (left) and $(j, k)=(2,1)$ (right). Using Theorem 1.4 for the unbounded component and Theorem 1.5(b) along with the numerically established fact that $[1 /(a-\lambda)]_{k-j}$ is nowhere locally constant, we can accept Figure 4 as a good approximation to $\operatorname{sp}_{7}^{(j, k)} L(a)$.

Figures 5 through 8 investigate a different symbol, which we call the capricorn symbol (

$$
\begin{aligned}
a(t)= & (-0.69-0.13 i) z^{-5}+(0.73-0.52 i) z^{-4}+(-0.06-0.74 i) z^{-3} \\
& +(-0.31-0.04 i) z^{-2}+(-0.11+0.10 i) z^{-1}+(-2.26+1.57 i) z \\
& +(-0.13+0.05 i) z^{2}+(0.53+0.21 i) z^{3}+(0.37-0.31 i) z^{4}+(0.22-0.03 i) z^{5}
\end{aligned}
$$

Figure 5 shows $a(\mathbf{T})$ and $B^{*}(a)$. Figure 6 is the analogue of Figure 2 and indicates the set

$$
\bigcup_{\substack{1 \leq j \leq 5 \\ 1 \leq k \leq 5}} \operatorname{sp}_{[-10,10]}^{(j, k)} L(a) .
$$

One can again numerically verify that $[1 /(a-\lambda)]_{k-j}$ is nowhere locally constant for $-5 \leq$ $k-j \leq 5$. Thus, Theorem 1.5(b) can be employed to justify that the antennae of Figure 6 are really all present in the set (7), and that Figures 7 and 8 should be good approximations to

$$
\mathrm{sp}_{10 \overline{\mathbf{D}}}^{(1,1)} L(a), \quad \mathrm{sp}_{10 \overline{\mathbf{D}}}^{(3,1)} L(a), \quad \mathrm{sp}_{10 \overline{\mathbf{D}}}^{(1,3)} L(a)
$$

Finally, Figure 9 involves tridiagonal matrices, illustrating the set

$$
\bigcup_{j-k \neq-1} \operatorname{sp}_{[-4,4]}^{(j, k)} L(a) \text { for } a(t)=t+t^{-1} / 9
$$

The limiting set (8) was derived in our paper [3] and is portrayed at the top of Figure 9; we believe this plot is correct to plotting accuracy. (Similar figures appear in [3].) Theorem 1.8 implies that

$$
\lim _{n \rightarrow \infty} \operatorname{sp}_{[-4,4]}^{(j, k)} C_{n}(a)=\mathrm{sp}_{[-4,4]}^{(j, k)} L(a)
$$

for the symbol under consideration, and Figure 9 illustrates this convincingly.

\section{Preliminaries}

Let $a \in \mathcal{P}$ and let $K$ be the infinite matrix that is supported in the sites $(j, k)$ with $j, k \in\{1, \ldots, m\}$. We denote by $P_{m}$ the projection that sends a sequence $x=\left\{x_{k}\right\}$ to the sequence given by $(P x)_{k}=x_{k}$ for $k \in\{1, \ldots, m\}$ and $(P x)_{k}=0$ otherwise. If $A=\left(a_{j k}\right)$ is an infinite matrix or a finite matrix of dimension $n \geq m$, we identify $P_{m} A P_{m}$ with the $m \times m$ matrix $\left(a_{j k}\right)_{j, k=1}^{m}$.

We know that $\operatorname{sp} L(a)=a(\mathbf{T})$. For $\lambda \in \mathbf{C} \backslash a(\mathbf{T})$, the inverse of $L(a)-\lambda I=L(a-\lambda)$ is $L\left((a-\lambda)^{-1}\right)$. The function

$$
f: \mathbf{C} \backslash a(\mathbf{T}) \rightarrow \mathbf{C}, \lambda \mapsto \operatorname{det}\left(I_{m}+P_{m} L\left((a-\lambda)^{-1}\right) P_{m} K P_{m}\right)
$$




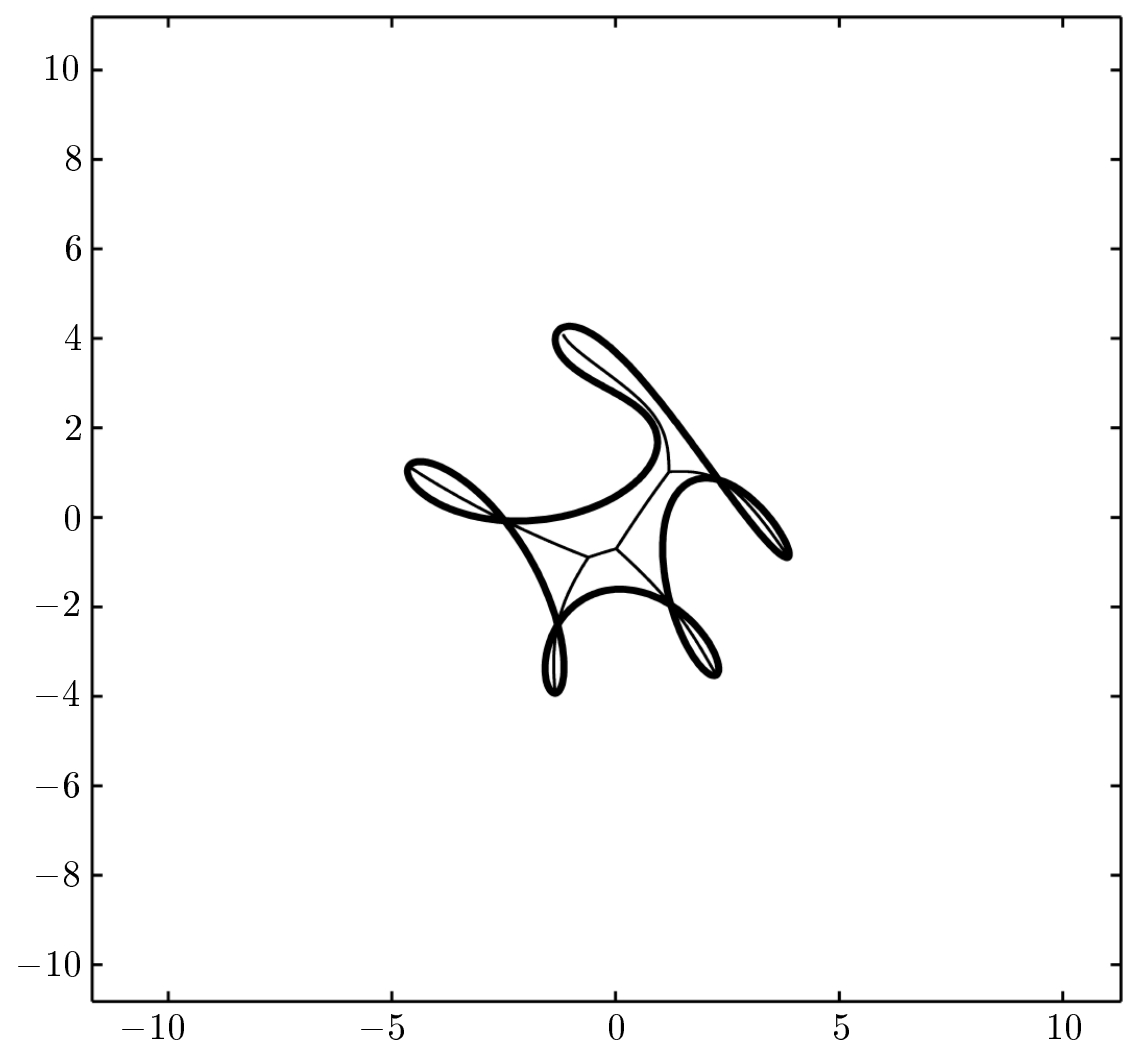

Figure 5: $a(\mathbf{T})$ (thick line) and $B^{*}(a)$ (thin line) for the capricorn symbol.

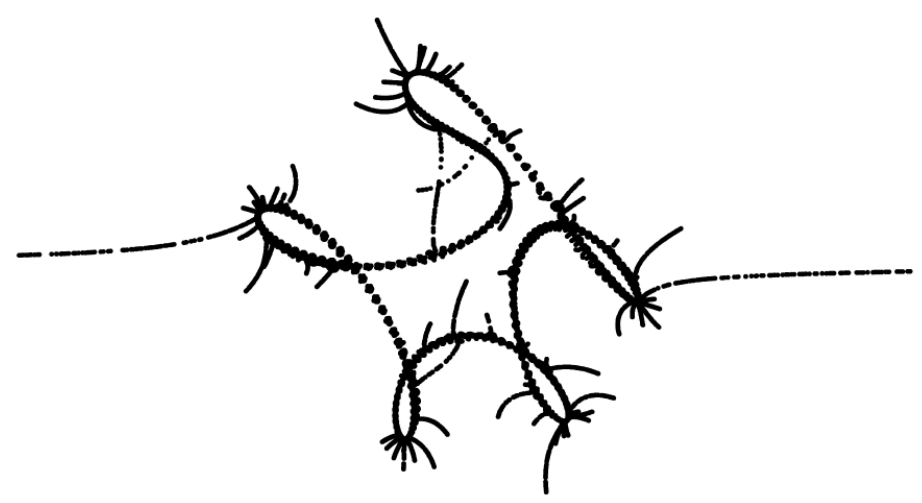

Figure 6: Eigenvalues of $C_{250}(a)$ for the capricorn symbol, perturbed in one randomly chosen entry in the upper left $5 \times 5$ block by a random number uniformly distributed in $[-10,10]$. This plot superimposes the eigenvalues of 2000 samples. 


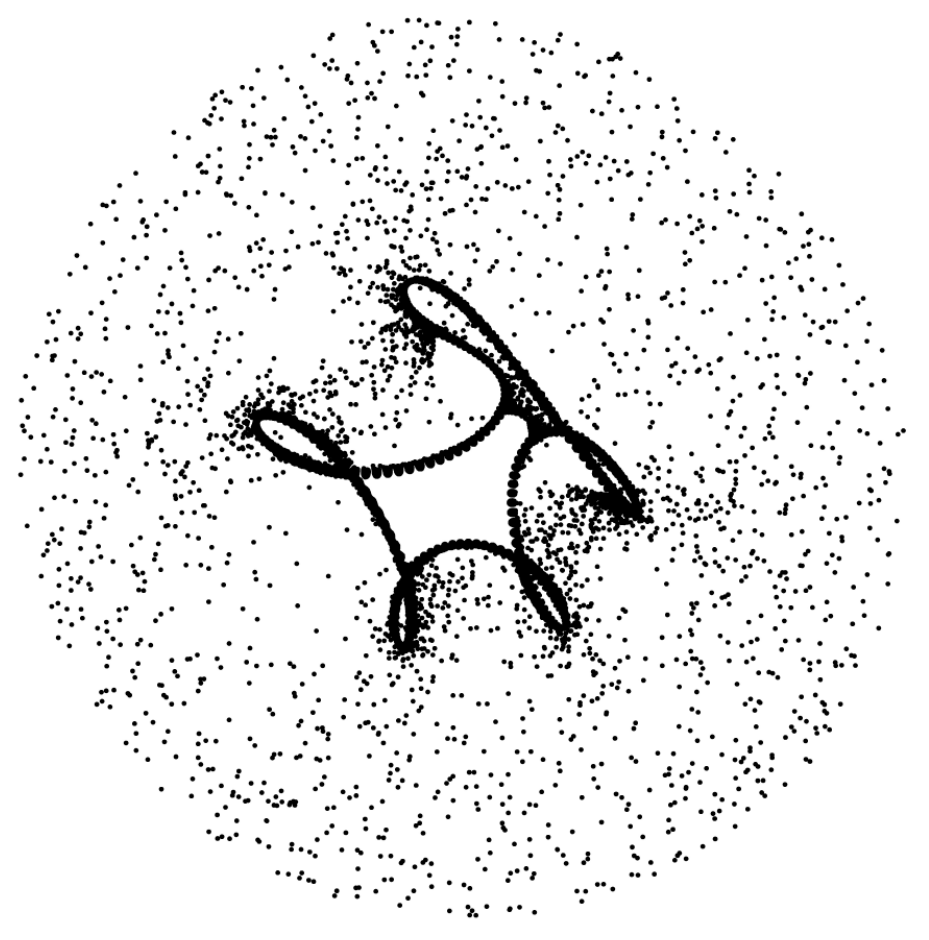

Figure 7: Eigenvalues of $C_{250}(a)$ for the capricorn symbol, perturbed in the $(j, j)$ entry by a random number uniformly distributed in $10 \overline{\mathbf{D}}$. This plot superimposes the eigenvalues of 2000 samples.
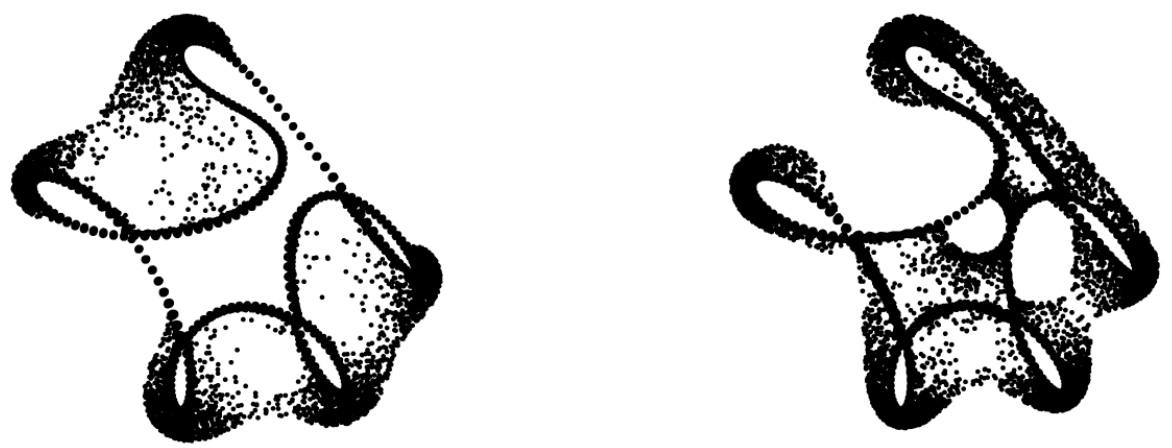

Figure 8: Eigenvalues of $C_{250}(a)$ for the capricorn symbol, perturbed in the $(j, k)$ entry by a random number uniformly distributed in $10 \overline{\mathbf{D}}$. On the left, $(j-k)(\bmod n)=-2$; on the right, $(j-k)(\bmod n)=2$. Each plot superimposes the eigenvalues of 2000 samples. 

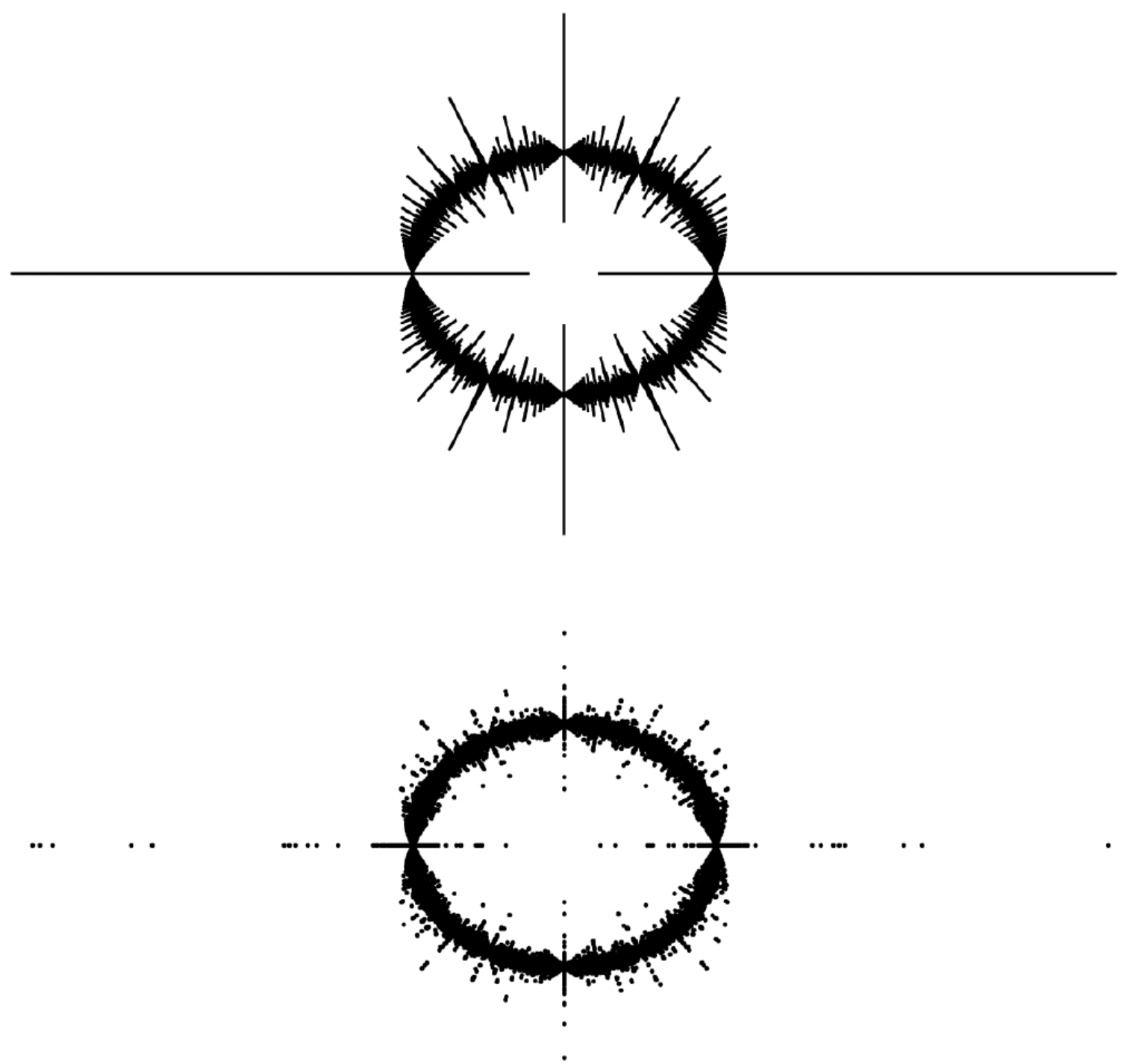

Figure 9: The set $\cup_{j \neq k-1} \operatorname{sp}_{[-4,4]}^{(j, k)} L(a)$ (top) and eigenvalues of random single entry perturbations to $C_{250}(a)$ from $[-4,4]$ (bottom) for the symbol $a(t)=t+\alpha^{2} t^{-1}$ with $\alpha=1 / 3$. (The lower plot superimposes the eigenvalues of 2000 samples.) 
is analytic, and it is easily checked that if $\lambda \in \mathbf{C} \backslash a(\mathbf{T})$, then

$$
\lambda \in \operatorname{sp}(L(a)+K) \Longleftrightarrow f(\lambda)=0 .
$$

We now state some well known facts for the circulant matrices $C_{n}(a)$; throughout what follows we assume that $n$ is at least as large as the bandwidth of $L(a)$. Let $w_{n}=e^{2 \pi i / n}$ and put

$$
F_{n}=\left(\begin{array}{ccccc}
1 & 1 & 1 & \cdots & 1 \\
1 & w_{n} & w_{n}^{2} & \cdots & w_{n}^{n-1} \\
1 & w_{n}^{2} & w_{n}^{4} & \cdots & w_{n}^{2(n-1)} \\
\vdots & \vdots & \vdots & & \vdots \\
1 & w_{n}^{n-1} & w_{n}^{2(n-1)} & \cdots & w_{n}^{(n-1)(n-1)}
\end{array}\right), \quad U_{n}=\frac{1}{\sqrt{n}} F_{n} .
$$

The matrix $U_{n}$ is unitary and a straightforward computation shows that

$$
C_{n}(a)-\lambda I_{n}=C_{n}(a-\lambda)=U_{n}^{*} \operatorname{diag}\left(a\left(w_{n}^{j}\right)-\lambda\right)_{j=0}^{n-1} U_{n} .
$$

Formula (11) implies that $\operatorname{sp} C_{n}(a)=a\left(\mathbf{T}_{n}\right)$. For $\lambda \notin a\left(\mathbf{T}_{n}\right)$, we denote by $C_{n}^{-1}(a-\lambda)$ the inverse of $C_{n}(a-\lambda)$. By analogy to (9) and (10), we see that the functions

$$
f_{n}: \mathbf{C} \backslash a\left(\mathbf{T}_{n}\right) \rightarrow \mathbf{C}, \lambda \mapsto \operatorname{det}\left(I_{m}+P_{m} C_{n}^{-1}(a-\lambda) P_{m} K P_{m}\right)
$$

are analytic and that, for $\lambda \in \mathbf{C} \backslash a\left(\mathbf{T}_{n}\right)$,

$$
\lambda \in \operatorname{sp}\left(C_{n}(a)+P_{n} K P_{n}\right) \Longleftrightarrow f_{n}(\lambda)=0 .
$$

Moreover, formula (11) gives

$$
C_{n}^{-1}(a-\lambda)=U_{n}^{*} \operatorname{diag}\left(\left(a\left(w_{n}^{j}\right)-\lambda\right)^{-1}\right)_{j=0}^{n-1} U_{n} .
$$

and hence the $(j, k)$ entry of $C_{n}^{-1}(a-\lambda)$ is

$$
\left[C_{n}^{-1}(a-\lambda)\right]_{j k}=\frac{1}{n} \sum_{\ell=0}^{n-1} \frac{\bar{w}_{n}^{\ell(j-1)} w_{n}^{\ell(k-1)}}{a\left(w_{n}^{\ell}\right)-\lambda} .
$$

For $\lambda \notin a(\mathbf{T})$, the right-hand side of (15) converges to

$$
\frac{1}{2 \pi} \int_{0}^{2 \pi} \frac{e^{-i \theta(j-k)}}{a\left(e^{i \theta}\right)-\lambda} d \theta=\left((a-\lambda)^{-1}\right)_{j-k}
$$

and the convergence is uniform on compact subsets of $\mathbf{C} \backslash a(\mathbf{T})$. It follows that

$$
\lim _{n \rightarrow \infty}\left[C_{n}^{-1}(a-\lambda)\right]_{j k}=\left[L\left((a-\lambda)^{-1}\right)\right]_{j k}
$$

and thus

$$
\lim _{n \rightarrow \infty} f_{n}(\lambda)=f(\lambda),
$$

the convergence being uniform on compact subsets of $\mathbf{C} \backslash a(\mathbf{T})$. 
Theorem 3.1 (Hurwitz). Let $G \subset \mathbf{C}$ be an open set, let $f$ be a function that is analytic in $G$ and does not vanish identically, and let $\left\{f_{n}\right\}$ be a sequence of analytic functions in $G$ that converges to $f$ uniformly on compact subsets of $G$. If $f(\lambda)=0$ for some $\lambda \in G$, then there is a sequence $\left\{\lambda_{n}\right\}$ of points $\lambda_{n} \in G$ such that $\lambda_{n} \rightarrow \lambda$ as $n \rightarrow \infty$ and $f_{n}\left(\lambda_{n}\right)=0$ for all sufficiently large $n$.

Two proofs of Hurwitz' theorem are in [18, pp. 205 and 312], for example.

Let $\left\{M_{n}\right\}$ be a sequence of nonempty subsets of $\mathbf{C}$. The uniform limiting set $\lim \inf M_{n}$ is defined as the set of all $\lambda \in \mathbf{C}$ that are the limit of some sequence $\left\{\lambda_{n}\right\}$ with $\lambda_{n} \in M_{n}$, while the partial limiting set $\lim \sup M_{n}$ is the set of all $\lambda \in \mathbf{C}$ that are a partial limit of some sequence $\left\{\lambda_{n}\right\}$ with $\lambda_{n} \in M_{n}$. Naturally, $\lim \inf M_{n} \subset \lim \sup M_{n}$.

Theorem 3.2 (Hausdorff). Let $M$ and the members of the sequence $\left\{M_{n}\right\}$ be nonempty compact subsets of $\mathbf{C}$. Then $\left\{M_{n}\right\}$ converges to $M$ in the Hausdorff metric,

$$
\lim _{n \rightarrow \infty} M_{n}=M
$$

if and only if

$$
\liminf _{n \rightarrow \infty} M_{n}=\limsup _{n \rightarrow \infty} M_{n}=M
$$

Proofs can be found in [9, Sections 3.1.1 and 3.1.2] and in [11, Section 2.8].

\section{Deterministic perturbations}

This section is devoted to the proofs of Theorems 1.1 and 1.3.

Proof of Theorem 1.1. We abbreviate $L(a)+K$ and $C_{n}(a)+P_{n} K P_{n}$ to $A$ and $A_{n}$, respectively. Since the connected component $G$ is not a subset of $\operatorname{sp} A$, we infer from (10) that the function $f$ given by (9) does not vanish identically on $G$. Define $f_{n}$ by (12).

We first show that $\lim \sup \left(\left(\operatorname{sp} A_{n} \cap G\right) \cup \partial G\right) \subset \operatorname{sp} A \cap \bar{G}$. Suppose $\lambda$ is not in $\operatorname{sp} A \cap \bar{G}$. If $\lambda \notin G$, then $\lambda$ is clearly not in the partial limiting set. Thus, let $\lambda \in G$. As $\lambda \notin \operatorname{sp} A$, we see that $f(\lambda) \neq 0$ due to (10). From (17) we therefore conclude that there is an open neighborhood $U \subset G$ of $\lambda$ and a natural number $n_{0}$ such that $f_{n}(\mu) \neq 0$ for all $\mu \in U$ and all $n \geq n_{0}$. Consequently, by (13), $U \cap \operatorname{sp} A_{n}=\emptyset$ for all $n \geq n_{0}$, which implies that $\lambda$ is not in $\lim \sup \left(\left(\operatorname{sp} A_{n} \cap G\right) \cup \partial G\right)$.

We now prove that $\operatorname{sp} A \cap \bar{G} \subset \liminf \left(\left(\operatorname{sp} A_{n} \cap G\right) \cup \partial G\right)$. Pick $\lambda$ in sp $A \cap \bar{G}$. If $\lambda \in \partial G$, then $\lambda$ is obviously in the uniform limiting set. So assume $\lambda \in G$. Then $f(\lambda)=0$ by virtue of (10). Hence, Theorem 3.1 guarantees the existence of $\lambda_{n} \in G$ such that $\lambda_{n} \rightarrow \lambda$ and $f_{n}\left(\lambda_{n}\right)=0$ for all sufficiently large $n$. From (13) we infer that $\lambda_{n} \in \operatorname{sp} A_{n}$. It follows that $\lambda$ belongs to $\lim \inf \left(\left(\operatorname{sp} A_{n} \cap G\right) \cup \partial G\right)$.

Combining the two inclusions shown in the preceding two paragraphs with Theorem 3.2, we arrive at equality (1). 
Example 4.1. Here we demonstrate that the hypothesis on $G$ in Theorem 1.1 is necessary in general; that is, we show that the equality (1) does not generally hold if the connected component $G$ of $\mathbf{C} \backslash a(\mathbf{T})$ is entirely contained in the set $\operatorname{sp}(L(a)+K)$. Let $a(t)=$ $t+\alpha^{2} t^{-1}(t \in \mathbf{T})$ with $\alpha \in[0,1]$. The set $a(\mathbf{T})$ is the ellipse

$$
\left\{\left(1+\alpha^{2}\right) \cos \theta+i\left(1-\alpha^{2}\right) \sin \theta: 0 \leq \theta<2 \pi\right\},
$$

with foci $\pm 2 \alpha$. Let $G_{+}$and $G_{-}$denote the bounded and unbounded components of $\mathbf{C} \backslash a(\mathbf{T})$ respectively. Notice that $a(\mathbf{T})=\mathbf{T}$ if $\alpha=0$ and $a(\mathbf{T})=[-2,2]$ (whence $G_{+}=\emptyset$ ) if $\alpha=1$. Theorem 1.1 shows that

$$
\lim _{n \rightarrow \infty}\left(\left(\operatorname{sp}\left(C_{n}(a)+P_{n} K P_{n}\right) \cap G_{-}\right) \cup a(\mathbf{T})\right)=\operatorname{sp}(L(a)+K) \cap \bar{G}_{-} .
$$

In particular, it follows that (2) holds for $\alpha=1$ and an arbitrary finitely-supported matrix $K$. We therefore consider the case where $\alpha \in[0,1)$ and $\lambda \in G_{+}$.

First let $\alpha=0$. Then $G_{+}=\mathbf{D}=\{\lambda \in \mathbf{C}:|\lambda|<1\}$, and it is easily seen that

$$
P_{m} L\left((a-\lambda)^{-1}\right) P_{m}=\left(\begin{array}{ccccc}
0 & 1 & \lambda & \lambda^{2} & \ldots \\
0 & 0 & 1 & \lambda & \ldots \\
0 & 0 & 0 & 1 & \ldots \\
0 & 0 & 0 & 0 & \ldots \\
\cdots & \ldots & \ldots & \ldots & \ldots
\end{array}\right) .
$$

Formula (15) and a straightforward computation give

$$
C_{n}^{-1}(a-\lambda)=\frac{1}{1-\lambda^{n}}\left(\begin{array}{ccccc}
\lambda^{n-1} & 1 & \lambda & \cdots & \lambda^{n-2} \\
\lambda^{n-2} & \lambda^{n-1} & 1 & \cdots & \lambda^{n-3} \\
\lambda^{n-3} & \lambda^{n-2} & \lambda^{n-1} & \cdots & \lambda^{n-4} \\
\vdots & \vdots & \vdots & & \vdots \\
1 & \lambda & \lambda^{2} & \cdots & \lambda^{n-1}
\end{array}\right)
$$

Let $E_{j} \omega E_{k}$ be the matrix whose $(j, k)$ entry is $\omega$ with all other entries zero. From (10) we observe that $\lambda \in \operatorname{sp}\left(L(a)+E_{j} \omega E_{k}\right)$ if and only if $1+\left[L\left((a-\lambda)^{-1}\right)\right]_{k j} \omega=0$. Taking into account (19) we see that $\mathbf{D}$ is contained in $\operatorname{sp}\left(L(a)+E_{j} \omega E_{k}\right)$ only when $j-k=1$ and $\omega=-1$. In that case (13) and (20) imply that $\operatorname{sp}\left(C_{n}(a)+E_{j} \omega E_{k}\right) \cap \mathbf{D}$ is $\{0\}$.

Now let $\alpha \in(0,1)$. Every point $\lambda \in G_{+}$can be written in the form

$$
\lambda=\rho e^{i \theta}+\alpha^{2} \rho^{-1} e^{-i \theta} \quad \text { with } \quad \alpha \leq \rho<1,0 \leq \theta<2 \pi .
$$

It follows that $a(t)-\lambda=t^{-1}\left(t-z_{1}\right)\left(t-z_{2}\right)$ with $z_{1}=\alpha^{2} \rho^{-1} e^{-i \theta}$ and $z_{2}=\rho e^{i \theta}$. As $\left|z_{1}\right|<1$ and $\left|z_{2}\right|<1$, we get

$$
\frac{1}{a(t)-\lambda}=\frac{1}{t}\left(1+\frac{z_{1}}{t}+\frac{z_{1}^{2}}{t^{2}}+\cdots\right)\left(1+\frac{z_{2}}{t}+\frac{z_{2}^{2}}{t^{2}}+\cdots\right)
$$

whence

$$
P_{m} L\left((a-\lambda)^{-1}\right) P_{m}=\left(\begin{array}{ccccc}
0 & 1 & z_{1}+z_{2} & z_{1}^{2}+z_{1} z_{2}+z_{2}^{2} & \cdots \\
0 & 0 & 1 & z_{1}+z_{2} & \cdots \\
0 & 0 & 0 & 1 & \cdots \\
0 & 0 & 0 & 0 & \cdots \\
\cdots & \ldots & \cdots & \cdots & \cdots
\end{array}\right)
$$


Notice that $z_{1}+z_{2}=\lambda$ and $z_{1}^{2}+z_{1} z_{2}+z_{2}^{2}=\lambda^{2}-\alpha^{2}$. The values of $\left[L\left((a-\lambda)^{-1}\right)\right]_{k j}$ at $\lambda=0$ and $\lambda=2 \alpha$ are easily seen to be different if $j-k \geq 2$. From (9) we therefore get that $G_{+}$ is a subset of $\operatorname{sp}\left(L(a)+E_{j} \omega E_{k}\right)$ if and only if $j-k=1$ and $\omega=-1$. So let $j-k=1$ and $\omega=-1$. By $(13)$,

$$
M_{n}:=\operatorname{sp}\left(C_{n}(a)+E_{j} \omega E_{k}\right) \cap G_{+}=\left\{\lambda \in G_{+}: 1-\left[C_{n}^{-1}(a-\lambda)\right]_{k j}=0\right\},
$$

and from (15) and (22) we conclude that

$$
\begin{aligned}
{\left[C_{n}^{-1}(a-\lambda)\right]_{k j}=} & \frac{1}{n} \sum_{\ell=0}^{n-1}\left(1+\frac{z_{1}}{w_{n}^{\ell}}+\frac{z_{1}^{2}}{w_{n}^{2 \ell}}+\cdots\right)\left(1+\frac{z_{2}}{w_{n}^{\ell}}+\frac{z_{2}^{2}}{w_{n}^{2 \ell}}+\cdots\right) \\
= & 1+\left(z_{1}^{n}+z_{1}^{n-1} z_{2}+\cdots+z_{2}^{n}\right) \\
& +\left(z_{1}^{2 n}+z_{1}^{2 n-1} z_{2}+\cdots+z_{2}^{2 n}\right)+\cdots \\
= & 1+\frac{z_{1}^{n+1}-z_{2}^{n+1}}{z_{1}-z_{2}}+\frac{z_{1}^{2 n+1}-z_{2}^{2 n+1}}{z_{1}-z_{2}}+\cdots \\
= & 1+\frac{1}{z_{1}-z_{2}}\left(\frac{z_{1}^{n+1}}{1-z_{1}^{n}}-\frac{z_{2}^{n+1}}{1-z_{2}^{n}}\right) .
\end{aligned}
$$

Recalling that $z_{1}=\alpha^{2} \rho^{-1} e^{-i \theta}$ and $z_{2}=\rho e^{i \theta}$, we see that the set $(24)$ is

$$
M_{n}=\left\{\lambda \in G_{+}: \rho^{2 n+2} e^{i(2 n+2) \theta}=\left(1-\rho^{n} e^{i n \theta}\right) \alpha^{2 n+2}+\alpha^{2 n} \rho^{n+2} e^{i(n+2) \theta}\right\} .
$$

Hence, if $\lambda \in M_{n}$, then

$$
\alpha^{2 n+2} \leq \rho^{2 n+2}<2 \alpha^{2 n+2}+\alpha^{2 n}<3 \alpha^{2 n} .
$$

It follows that $\rho \rightarrow \alpha$ as $n \rightarrow \infty$, and so (21) implies that $\lim \sup M_{n} \subset[-2 \alpha, 2 \alpha]$. In other words, the sets $(24)$ cluster on the segment between the foci of the ellipse $G_{+}$and do not fill out all of $G_{+}$.

To physicists, the matrix $C_{n}(a)$ is the deterministic component of a model for "a quantum particle hopping on a ring", in the language of Feinberg and Zee [6]. Each row of $C_{n}(a)$ corresponds to one of $n$ sites arranged in a circle, each one of which only communicates with its nearest neighbors on either side. The perturbation $E_{j} \omega E_{k}$ (for $j-k=1$ and $\omega=-1$ ) essentially fractures this periodic structure in the more dominant direction. For $n>2$, the resulting matrix is unitarily similar to the Toeplitz matrix $T_{n}\left(a_{n}\right)$ for $a_{n}(t)=t+\alpha^{2}\left(t^{-1}+t^{n}\right)$; its eigenvalues cluster on $[-2 \alpha, 2 \alpha]$, but are exceptionally sensitive to perturbations (see [2], [15], [17]). Thus, the rather benign-looking perturbation $E_{j} \omega E_{k}$ transforms a perfectly-conditioned normal matrix eigenvalue problem into a dramatically ill-conditioned one.

Proof of Theorem 1.2. By virtue of Theorem 1.1 and (10), it suffices to prove that the function $f$ defined by $(9)$ is not identically zero in $G$.

(a) Pick $\lambda \in G \backslash B^{*}(a)$. Then there exists a $\rho>0$ such that $\lambda \notin B\left(a_{\rho}\right)$. Put $D_{\rho}=$ $\operatorname{diag}\left(1, \rho, \ldots, \rho^{m-1}\right)$. One can directly verify that

$$
P_{m} L\left((a-\lambda)^{-1}\right) P_{m}=D_{\rho}^{-1} P_{m} L\left(\left(a_{\rho}-\lambda\right)^{-1}\right) P_{m} D_{\rho},
$$


and since $K$ is diagonal, we get

$$
\begin{aligned}
P_{m} L\left((a-\lambda)^{-1}\right) P_{m} K P_{m} & =D_{\rho}^{-1} P_{m} L\left(\left(a_{\rho}-\lambda\right)^{-1}\right) P_{m} D_{\rho} K P_{m} \\
& =D_{\rho}^{-1} P_{m} L\left(\left(a_{\rho}-\lambda\right)^{-1}\right) P_{m} K P_{m} D_{\rho} .
\end{aligned}
$$

Consequently,

$$
\begin{aligned}
f(\lambda) & =\operatorname{det}\left(I_{m}+P_{m} L\left((a-\lambda)^{-1}\right) P_{m} K P_{m}\right) \\
& =\operatorname{det}\left(I_{m}+P_{m} L\left(\left(a_{\rho}-\lambda\right)^{-1}\right) P_{m} K P_{m}\right) .
\end{aligned}
$$

The determinant (26) is 1 at $\lambda=\infty$, and hence it cannot vanish identically in the unbounded component of $\mathbf{C} \backslash a_{\rho}(\mathbf{T})$. Because $\lambda \in \mathbf{C} \backslash B\left(a_{\rho}\right)$ and $\mathbf{C} \backslash B\left(a_{\rho}\right)$ is the unbounded component of $\mathbf{C} \backslash a_{\rho}(\mathbf{T})$, it follows that $f$ is not identically zero in an open neighborhood of $\lambda$ and thus not identically zero throughout $G$.

(b) In this case, the function (9) is $f(\lambda)=1+\left[(a-\lambda)^{-1}\right]_{k-j} \omega$, and our assumption clearly implies that $f$ cannot be identically zero in $G$.

Proof of Corollary 1.3. From Example 4.1 we know that the ranges $a_{\rho}(\mathbf{T})$ are ellipses with the same foci $\pm 2 \alpha$, and $B^{*}(a)$ is seen to be simply the line segment between the foci. Since each component of $\mathbf{C} \backslash a(\mathbf{T})$ certainly contains a point outside this line segment, Theorem 1.2 implies that

$$
\lim _{n \rightarrow \infty}\left(\left(\operatorname{sp}\left(C_{n}(a)+P_{n} K P_{n}\right) \cap G_{ \pm}\right) \cup a(\mathbf{T})\right)=\operatorname{sp}(L(a)+K) \cap \bar{G}_{ \pm} .
$$

Because $\lim \left(X_{n} \cup Y_{n}\right)=\lim X_{n} \cup \lim Y_{n}$ (which easily follows from Theorem 3.2), we arrive at $(2)$.

\section{Random perturbations}

In this section we give proofs to Theorems 1.4, 1.5, 1.7, and 1.8, as well as Corollary 1.6. Throughout what follows, $E$ is a finite set of sites $(j, k)$ with $j, k \in\{1, \ldots, m\}$ and $\Omega=$ $\left\{\Omega_{j k}\right\}_{(j, k) \in E}$ is a family of compact subsets of $\mathbf{C}$, each containing the origin.

Proposition 5.1 If $G$ is a connected component of $\mathbf{C} \backslash a(\mathbf{T})$, then

$$
\limsup _{n \rightarrow \infty}\left(\operatorname{sp}_{\Omega}^{E} C_{n}(a) \cap \bar{G}\right) \subset \operatorname{sp}_{\Omega}^{E} L(a) \cap \bar{G} .
$$

Proof. Pick $\lambda$ in the left-hand side of (27). If $\lambda \in \partial G$, then $\lambda$ is in the right-hand side of (27) because $a(\mathbf{T}) \subset \operatorname{sp} L(a) \subset \operatorname{sp}_{\Omega}^{E} L(a)$. So assume that $\lambda \in G$. By the definition of the partial limiting set, there are $\lambda_{n_{\ell}} \in \operatorname{sp}_{\Omega}^{E} C_{n_{\ell}}(a) \cap G$ such that $\lambda_{n_{\ell}} \rightarrow \lambda$. From (3) and (13) we infer that for each $n_{\ell}$ there exists $K_{n_{\ell}} \in \mathcal{K}_{\Omega}^{E}$ such that

$$
\operatorname{det}\left(I_{m}+P_{m} C_{n_{\ell}}^{-1}\left(a-\lambda_{n_{\ell}}\right) P_{m} K_{n_{\ell}} P_{m}\right)=0 .
$$

Since $\mathcal{K}_{\Omega}^{E}$ is a compact set, the matrices $K_{n_{\ell}}$ have a partial limit $K$ in $\mathcal{K}_{\Omega}^{E}$. From (16) and (28) we therefore get

$$
\operatorname{det}\left(I_{m}+P_{m} L\left((a-\lambda)^{-1}\right) P_{m} K P_{m}\right)=0
$$


which, by (3) and (10), shows that $\lambda$ is in the right-hand side of (27).

Proof of Theorem 1.4. By virtue of Proposition 5.1 it suffices to show that

$$
\operatorname{sp}_{\Omega}^{E} L(a) \cap \bar{G} \subset \liminf _{n \rightarrow \infty}\left(\operatorname{sp}_{\Omega}^{E} C_{n}(a) \cap \bar{G}\right) .
$$

Since $a\left(\mathbf{T}_{n}\right)=\operatorname{sp} C_{n}(a) \subset \operatorname{sp}_{\Omega}^{E} C_{n}(a)$, a point $\lambda \in \partial G$ certainly belongs to the right-hand side of (29). Thus, let $\lambda \in \operatorname{sp}_{\Omega}^{E} L(a) \cap G$. There exists a $K \in \mathcal{K}_{\Omega}^{E}$ such that $\lambda \in \operatorname{sp}(L(a)+K)$. By assumption, the component $G$ is not contained in $\operatorname{sp}_{\Omega}^{E} L(a)$, and hence it cannot be a subset of $\operatorname{sp}(L(a)+K)$. Thus, we can apply Theorem 1.1 to conclude that there exist $\lambda_{n} \in \operatorname{sp}\left(C_{n}(a)+P_{n} K P_{n}\right) \cap G \subset \operatorname{sp}_{\Omega}^{E} C_{n}(a) \cap G$ such that $\lambda_{n} \rightarrow \lambda$, which implies that $\lambda$ is in the right-hand side of (29).

Proof of Theorem 1.5. Again, we are left to prove inclusion (29). If $\lambda \in \partial G$, then $\lambda$ is in $\lim \inf a\left(\mathbf{T}_{n}\right)$ and hence in the right-hand side of (29). Thus, let $\lambda \in \operatorname{sp}_{\Omega}^{E} L(a) \cap G$. There is a $K \in \mathcal{K}_{\Omega}^{E}$ such that $\lambda \in \operatorname{sp}(L(a)+K) \cap G$. From Theorem 1.2 we deduce that there exist $\lambda_{n} \in \operatorname{sp}\left(C_{n}(a)+P_{n} K P_{n}\right) \cap G \subset \operatorname{sp}_{\Omega}^{E} C_{n}(a) \cap \bar{G}$ such that $\lambda_{n} \rightarrow \lambda$. Consequently, $\lambda$ belongs to the right-hand side of (29).

Proof of Corollary 1.6. This is immediate from Theorem 1.5, because $B^{*}(a)$ is the line segment $[-2 \alpha, 2 \alpha]$.

Put $\Omega^{\circ}=\left\{\Omega_{j k}^{\circ}\right\}_{(j, k) \in E}$, where $\Omega_{j k}^{\circ}$ is the set of interior points of $\Omega_{j k}$. We define

$$
\operatorname{sp}_{\Omega^{\circ}}^{E} A=\bigcup_{K \in \mathcal{K}_{\Omega^{\circ}}^{E}} \operatorname{sp}(A+K)
$$

if none of the sets $\Omega_{j k}^{\circ}$ is empty, and we let $\operatorname{sp}_{\Omega^{\circ}}^{E} A=\emptyset$ if one of the sets $\Omega_{j k}^{\circ}$ is the empty set.

Proposition 5.2 If $G$ is a connected component of $\mathbf{C} \backslash a(\mathbf{T})$, then

$$
\operatorname{sp}_{\Omega^{\circ}}^{E} L(a) \cap \bar{G} \subset \liminf _{n \rightarrow \infty}\left(\operatorname{sp}_{\Omega}^{E} C_{n}(a) \cap \bar{G}\right) .
$$

Proof. Let $\lambda \in \operatorname{sp}_{\Omega^{\circ}}^{E} L(a) \cap \bar{G}$. As in the proof of Theorem 1.4, we may suppose that $\lambda \in G$. By (10) and (30), there exists a $K \in \mathcal{K}_{\Omega^{\circ}}^{E}$ such that

$$
\operatorname{det}\left(I_{m}+P_{m} L\left((a-\lambda)^{-1}\right) P_{m} K P_{m}\right)=0 .
$$

We define the entire functions $\varphi$ and $\varphi_{n}$ by

$$
\begin{aligned}
\varphi(z) & =\operatorname{det}\left(I_{m}+P_{m} L\left((a-\lambda)^{-1}\right) P_{m}(K-z K) P_{m}\right), \\
\varphi_{n}(z) & =\operatorname{det}\left(I_{m}+P_{m} C_{n}^{-1}(a-\lambda) P_{m}(K-z K) P_{m}\right) .
\end{aligned}
$$

From (16) we see that $\varphi_{n}$ converges to $\varphi$ uniformly on compact subsets of C. As $\varphi(1)=1$, the function $\varphi$ is not identically zero. Since $\varphi(0)=0$, we deduce from Theorem 3.1 that there are $z_{n} \in \mathbf{C}$ such that $z_{n} \rightarrow 0$ and $\varphi_{n}\left(z_{n}\right)=0$ for all sufficiently large $n$. Consequently, by (13), $\lambda \in \operatorname{sp}\left(C_{n}(a)+P_{n}\left(K-z_{n} K\right) P_{n}\right)$. As $K-z_{n} K \in \mathcal{K}_{\Omega^{\circ}}^{E}$ whenever $z_{n}$ is sufficiently 
close to zero, it follows that $\lambda \in \operatorname{sp}_{\Omega^{\circ}}^{E} C_{n}(a)$ for all $n$ large enough and hence that $\lambda$ is in the right-hand side of (31).

Proof of Theorem 1.7. Equality (5) is true for some $\varepsilon \in(0, \infty)$ if (and only if)

$$
\lim _{n \rightarrow \infty}\left(\operatorname{sp}_{\varepsilon \Omega}^{E} C_{n}(a) \cap \bar{G}\right)=\operatorname{sp}_{\varepsilon \Omega}^{E} L(a) \cap \bar{G}
$$

for every connected component $G$ of $\mathbf{C} \backslash a(\mathbf{T})$. Theorem 1.4 shows that $(32)$ is true if $G$ is the unbounded component. We now prove that for each bounded component $G$ there is at most one value $\varepsilon(G)$ for which (32) is not valid. This will imply the assertion of Theorem 1.7.

By virtue of Proposition 5.1, equality (32) will follow as soon as we have shown that $\operatorname{sp}_{\varepsilon \Omega}^{E} L(a) \cap \bar{G} \subset \liminf _{n \rightarrow \infty}\left(\operatorname{sp}_{\varepsilon \Omega}^{E} C_{n}(a) \cap \bar{G}\right)$. Furthermore, since $a\left(\mathbf{T}_{n}\right)=\operatorname{sp} C_{n}(a) \subset$ $\operatorname{sp}_{\varepsilon \Omega}^{E} C_{n}(a)$, it suffices to show that

$$
\operatorname{sp}_{\varepsilon \Omega}^{E} L(a) \cap G \subset \liminf _{n \rightarrow \infty}\left(\operatorname{sp}_{\varepsilon \Omega}^{E} C_{n}(a) \cap \bar{G}\right) .
$$

Thus, let $G$ be a bounded component of $\mathbf{C} \backslash a(\mathbf{T})$. It is clear that $\operatorname{sp}_{\varepsilon \Omega}^{E} L(a)$ does not contain all of $G$ if $\varepsilon>0$ is sufficiently small. Put

$$
\varepsilon(G)=\sup \left\{\varepsilon>0: \operatorname{sp}_{\varepsilon \Omega}^{E} L(a) \text { does not contain } G\right\} .
$$

We claim that (33) holds for all $\varepsilon \neq \varepsilon(G)$.

Suppose $0<\varepsilon<\varepsilon(G)$. For $\lambda_{0} \in \operatorname{sp}_{\varepsilon \Omega}^{E} L(a) \cap G$, there is a $K \in \mathcal{K}_{\varepsilon \Omega}^{E}$ such that $f\left(\lambda_{0}\right)=0$, where $f$ is defined by (9). Since $G$ is not entirely contained in $\operatorname{sp}_{\varepsilon \Omega}^{E} L(a)$, we see from (10) that $f$ is not identically zero in $G$. Thus, proceeding as in the proof of Theorem 1.4, we conclude that $\lambda_{0}$ is in the right-hand side of (33).

Finally, suppose $\varepsilon>\varepsilon(G)$. In that case (33) amounts to the inclusion

$$
G \subset \liminf _{n \rightarrow \infty}\left(\operatorname{sp}_{\varepsilon \Omega}^{E} C_{n}(a) \cap \bar{G}\right) .
$$

Take any $\delta$ so that $\varepsilon(G)<\delta<\varepsilon$. Then $G \subset \operatorname{sp}_{\delta \Omega}^{E} L(a)$, and since each $\Omega_{j k}$ is starlike and the closure of its interior points, we have $\delta \Omega_{j k} \subset \varepsilon \Omega_{j k}^{\circ}$ and hence $\operatorname{sp}_{\delta \Omega}^{E} L(a) \subset \operatorname{sp}_{\varepsilon \Omega^{\circ}}^{E} L(a)$. Proposition 5.2 now gives (34).

Proof of Theorem 1.8. Recall the notation established in Example 4.1. We know from Theorem 1.7 that (32) is true for $G=G_{-}$and arbitrary compact sets $\Omega_{j k}$ containing the origin. By virtue of Proposition 5.1, equality (5) will follow once we have shown that

$$
\operatorname{sp}_{\varepsilon \Omega}^{E} L(a) \cap G_{+} \subset \liminf _{n \rightarrow \infty}\left(\operatorname{sp}_{\varepsilon \Omega}^{E} C_{n}(a) \cap G_{+}\right) .
$$

For $\alpha=1$, the left-hand side of (35) is empty. So let $\alpha \in[0,1)$. In Example 4.1, we showed that

$$
\begin{aligned}
\operatorname{sp}_{\varepsilon \Omega}^{E} L(a) \cap G_{+} & =\left\{\lambda \in G_{+}:\left[L\left((a-\lambda)^{-1}\right)\right]_{k j} \in-1 /\left(\varepsilon \Omega_{j k}\right)\right\}, \\
\operatorname{sp}_{\varepsilon \Omega}^{E} C_{n}(a) \cap G_{+} & =\left\{\lambda \in G_{+}:\left[C_{n}^{-1}(a-\lambda)\right]_{k j} \in-1 /\left(\varepsilon \Omega_{j k}\right)\right\},
\end{aligned}
$$


where $-1 /\left(\varepsilon \Omega_{j k}\right):=\left\{\lambda: 1+\lambda \omega=0\right.$ for some $\left.\omega \in \varepsilon \Omega_{j k}\right\}$. Pick $\lambda_{0}$ in (36). Then there exists some $\omega \in \varepsilon \Omega_{j k}$ such that the function $f(\lambda)=1+\omega\left[L\left((a-\lambda)^{-1}\right]_{k j}\right.$ vanishes at $\lambda_{0}$. By Example 4.1, $f$ is not identically zero in $G_{+}$whenever $j-k \neq 1$ or $\omega \neq-1$. Since $f_{n}(\lambda)=1+\omega\left[C_{n}^{-1}(a-\lambda)\right]_{k j}$ converges to $f(\lambda)$ uniformly on compact subsets of $G_{+}$by virtue of (16), we infer from Theorem 3.1 that if $j-k \neq 1$ or $\omega \neq-1$, then there exist $\lambda_{n} \in G_{+}$such that $\lambda_{n} \rightarrow \lambda_{0}$ and $f_{n}\left(\lambda_{n}\right)=0$ for all sufficiently large $n$. By (37), this means that $\lambda_{0}$ is in the right hand side of (35).

Now suppose $\alpha \in[0,1), j-k=1$, and $-1 \in \varepsilon \Omega_{j k}$. In this case (35) is equivalent to the inclusion

$$
G_{+} \subset \liminf _{n \rightarrow \infty}\left(\operatorname{sp}_{\varepsilon \Omega}^{E} C_{n}(a) \cap G_{+}\right) .
$$

Let $\gamma$ be a continuous curve between 0 and some point different from 0 such that $-1+\gamma \subset$ $\varepsilon \Omega_{j k}$. When $\alpha=0$, we have $G_{+}=\mathbf{D}$. We obtain from (20) and (37) that $\operatorname{sp}_{\varepsilon \Omega}^{E} C_{n}(a) \cap \mathbf{D}$ contains the set

$$
\left\{\lambda \in \mathbf{D}: 1+\omega /\left(1-\lambda^{n}\right)=0 \text { for some } \omega \in-1+\gamma\right\}=\left\{\lambda \in \mathbf{D}: \lambda^{n} \in \gamma\right\},
$$

and it is easily seen that the points of the latter set are asymptotically dense in $\mathbf{D}$ as $n \rightarrow \infty$, which proves (38).

Now let $\alpha \in(0,1)$. Then $(25)$ and (37) show that $\operatorname{sp}_{\varepsilon \Omega}^{E} C_{n}(a) \cap G_{+}$contains the set

$$
\left\{\lambda \in G_{+}: \frac{1}{z_{1}-z_{2}}\left(\frac{z_{1}^{n+1}}{1-z_{1}^{n}}-\frac{z_{2}^{n+1}}{1-z_{2}^{n}}\right) \in \delta\right\},
$$

where $\delta:=\{z /(1-z): z \in \gamma\}$. Clearly, $\delta$ is also a continuous curve between 0 and some point different from zero. Fix $\rho \in(\alpha, 1)$ and let $\lambda$ be of the form $(21)$. We then have

$$
\frac{1}{z_{1}-z_{2}}\left(\frac{z_{1}^{n+1}}{1-z_{1}^{n}}-\frac{z_{2}^{n+1}}{1-z_{2}^{n}}\right)=\frac{z_{2}^{n+1}}{z_{2}-z_{1}}\left(\frac{1}{1-z_{2}^{n}}-\left(\frac{z_{1}}{z_{2}}\right)^{n+1} \frac{1}{1-z_{1}^{n}}\right),
$$

and since, for sufficiently large $n$,

$$
\left|1-\frac{1}{1-z_{2}^{n}}\right|=\left|\frac{z_{2}^{n}}{1-z_{2}^{n}}\right| \leq 2 \rho^{n}, \quad\left|\frac{z_{1}}{z_{2}}\right|^{n+1}=\left(\frac{\alpha^{2}}{\rho^{2}}\right)^{n+1},
$$

it follows that as $n \rightarrow \infty$, (40) equals

$$
\frac{z_{2}^{n+1}}{z_{2}-z_{1}}\left(1+O\left(q^{n}\right)\right) \text { for some } q \in(0,1) \text {. }
$$

Consequently, the argument of (40) is

$$
\arg \left(\frac{e^{i(n+1) \theta}}{\alpha^{2} \rho^{-1} e^{-i \theta}-\rho e^{i \theta}}\left(1+O\left(q^{n}\right)\right)\right)=(n+1) \theta+\beta(\theta)+o(1),
$$

where $\beta$ is continuous and monotonically increasing on $[0,2 \pi)$ with $\beta(0+0)=\pi$ and $\beta(2 \pi-0)=5 \pi$. Thus, as $\lambda$ moves once counter-clockwise along the ellipse defined by (21), 
the point (40) traces out a small continuous curve around 0 (contained in a disk of radius $\left.O\left(\rho^{n+1}\right)\right)$ whose winding number with respect to $\theta$ is $n+3$. This curve intersects the curve $\delta$ at least $n+3$ times, and from (41) we see that the arguments $\theta$ of the $\lambda$ 's corresponding to the intersection points are asymptotically dense on $[0,2 \pi)$. As $\rho$ may be chosen arbitrarily in $(0,1)$, this proves that every point of $G_{+}$is in the uniform limiting set of the sets $(37)$ and hence implies (35).

Finally, let $\alpha \in[0,1), j-k=1$, and $\varepsilon \Omega_{j k}=\{-1,0\}$. From Example 4.1, together with (36) and (37), we infer that $\operatorname{sp}_{\varepsilon \Omega}^{E} L(a) \cap G_{+}=G_{+}$, while $\operatorname{sp}_{\varepsilon \Omega}^{E} C_{n}(a) \cap G_{+}$equals

$$
\begin{aligned}
\left\{\lambda \in G_{+}: \lambda^{n} \in\{-1,0\}\right\} & \text { for } \quad \alpha=0, \\
\left\{\lambda \in G_{+}: z_{1}^{n+1} /\left(1-z_{1}^{n}\right)=z_{2}^{n+1} /\left(1-z_{2}^{n}\right)\right\} & \text { for } \quad \alpha \in(0,1) .
\end{aligned}
$$

From (42) it is clear that (5) is not true for $\alpha=0$, and in Example 4.1 we showed that the points of $(43)$ cluster on $[-2 \alpha, 2 \alpha]$, revealing that (5) does not hold for $\alpha \in(0,1)$.

\section{$6 \quad$ Laurent versus Toeplitz}

The $n \times n$ Toeplitz matrix induced by $a \in \mathcal{P}$ is the matrix $T_{n}(a)=\left(a_{j-k}\right)_{j, k=1}^{n}$. Schmidt and Spitzer [19] showed that $\operatorname{sp} T_{n}(a)$ converges in the Hausdorff metric to some set $\Lambda(a)$ that is either a singleton or a finite union of analytic arcs. For example, if $a(t)=t+\alpha^{2} t^{-1}(t \in \mathbf{T})$ with $\alpha \in[0,1]$, then $\Lambda(a)=[-2 \alpha, 2 \alpha]$. For the $\&$ and with the set $B^{*}(a)$.

It turns out that in general $\operatorname{sp}_{\Omega}^{E} C_{n}(a)$ and $\operatorname{sp}_{\Omega}^{E} T_{n}(a)$ approach different limits as $n \rightarrow \infty$. This is nicely seen in Figure 10, where we compare real perturbations to the \& symbol for the circulant (Laurent) and Toeplitz cases.

\section{$7 \quad$ Some conjectures}

In this section we formulate a few conjectures that concern refinements of the results we have proved. Throughout what follows we suppose that $a \in \mathcal{P}$ and that $K$ has only finitely many nonzero entries.

Conjecture 7.1. We conjecture that perturbation of a banded Laurent matrix in a single entry of the main diagonal can never produce a spectrum that contains an entire component of the complement of the original spectrum, that is, we claim that $\operatorname{sp}\left(L(a)+E_{1} \omega E_{1}\right)$ never contains an entire component of $\mathbf{C} \backslash a(\mathbf{T})$. Since

$$
\operatorname{sp}\left(L(a)+E_{1} \omega E_{1}\right)=a(\mathbf{T}) \cup\left\{\lambda \notin a(\mathbf{T}): 1+\left[L\left((a-\lambda)^{-1}\right)\right]_{11} \omega=0\right\},
$$

this is equivalent to conjecturing that if $a \in \mathcal{P}$, then the zeroth Fourier coefficient of $(a-\lambda)^{-1}$ can never be a nonzero constant throughout some component of $\mathbf{C} \backslash a(\mathbf{T})$.

Conjecture 7.2. We conjecture that $\operatorname{sp}(L(a)+K) \backslash \operatorname{sp} L(a)$ is always the union of some components of $\mathbf{C} \backslash a(\mathbf{T})$ and a finite number of points. Equivalently, we conjecture that $\operatorname{sp}(L(a)+K) \backslash \operatorname{sp} L(a)$ is never countable. 

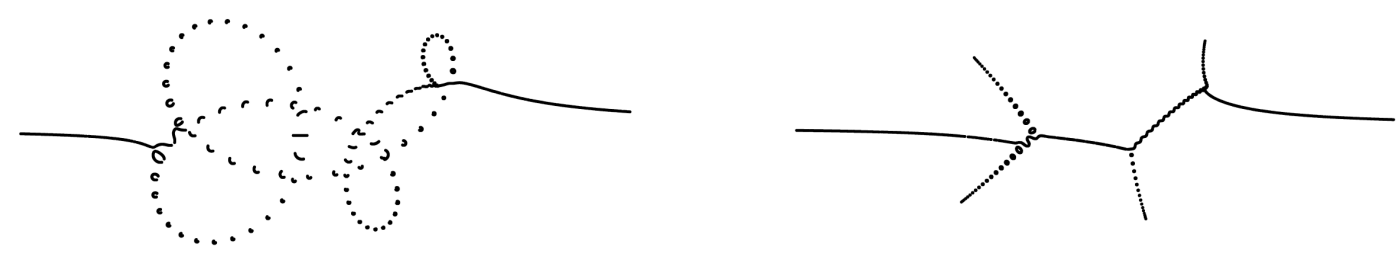

Real perturbations to the $(1,1)$ entry
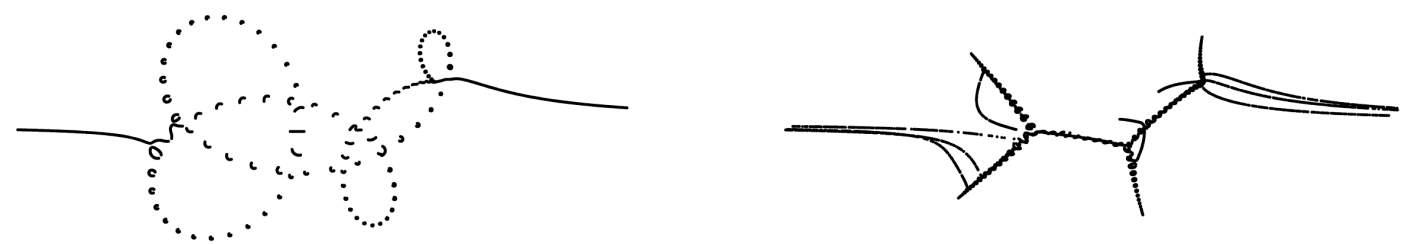

Real single entry perturbations to the $(j, j)$ entry for random $j \in\{1,2,3\}$
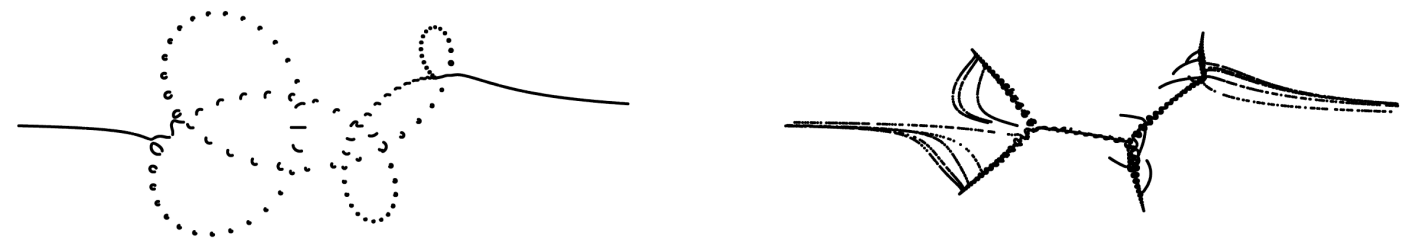

Real single entry perturbations to the $(j, j)$ entry for random $j \in\{1,2,3,4,5\}$
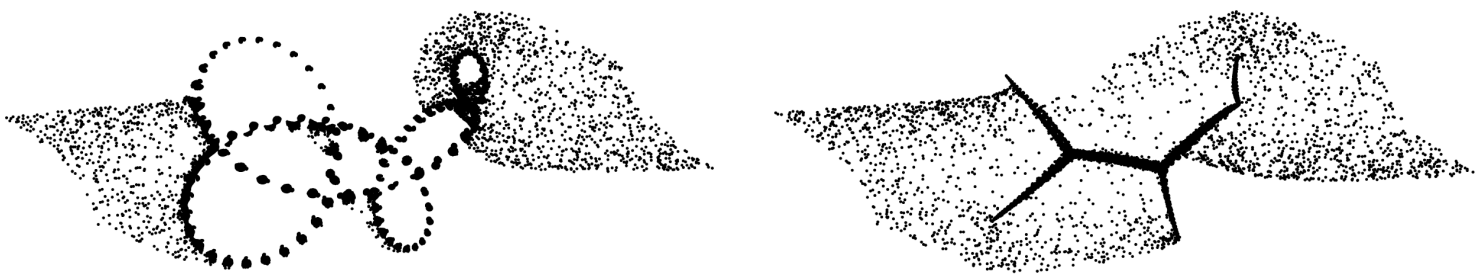

Simultaneous real perturbations to the $(1,1)$ and $(2,1)$ entries

Figure 10: Eigenvalues of random perturbations to $C_{100}(a)$ (left) and $T_{100}(a)$ (right). All perturbations are uniformly distributed in $[-7,7]$ and each plot superimposes the eigenvalues of 2000 samples. (The first three plots on the left are identical.) Note that the scale of these images differs from the one used in Figures 1-4. 
Conjecture 7.3. We conjecture that

$$
a(\mathbf{T}) \subset \liminf _{n \rightarrow \infty} \operatorname{sp}\left(C_{n}(a)+P_{n} K P_{n}\right)
$$

holds for all $a \in \mathcal{P}$, and that, in particular, (2) can be replaced by

$$
\lim _{n \rightarrow \infty} \operatorname{sp}\left(C_{n}(a)+P_{n} K P_{n}\right)=\operatorname{sp}(L(a)+K) .
$$

Conjecture 7.4. In connection with Theorem 1.1, we conjecture that if a bounded component $G$ of $\mathbf{C} \backslash a(\mathbf{T})$ is entirely contained in $\operatorname{sp}(L(a)+K)$, then

$$
\begin{aligned}
& \lim _{n \rightarrow \infty}\left(\left(\operatorname{sp}\left(C_{n}(a)+P_{n} K P_{n}\right) \cap G\right) \cup \partial G\right) \\
& \quad=\lim _{n \rightarrow \infty}\left(\left(\operatorname{sp} T_{n}(a) \cap G\right) \cup \partial G\right)=\Lambda(a) \cap \bar{G} .
\end{aligned}
$$

This conjecture includes the claim that (1) is never true if $G$ is a subset of $\operatorname{sp}(L(a)+K)$.

Conjecture 7.5. We conjecture that, under the hypothesis of Theorem 1.7, equality (5) is actually true for all $\varepsilon \in(0, \infty)$.

Conjecture 7.6. Let $\|\cdot\|$ be the operator norm on $\ell^{2}$. Given a bounded operator $A$ on $\ell^{2}$ or a matrix $A$, the structured pseudospectrum or spectral value set $\mathrm{sp}_{\varepsilon}^{m} A$ is defined by

$$
\mathrm{sp}_{\varepsilon}^{m} A=\bigcup_{\|K\| \leq \varepsilon} \operatorname{sp}\left(A+P_{m} K P_{m}\right)
$$

(see [3], [7], [12], [13]). In [7], it is shown that

$$
\operatorname{sp}_{\varepsilon}^{m} A=\operatorname{sp} A \cup\left\{\lambda \notin \operatorname{sp} A:\left\|P_{m}(A-\lambda I)^{-1} P_{m}\right\| \geq 1 / \varepsilon\right\} .
$$

Using this equality, we can show that if $a \in \mathcal{P}$, then

$$
\lim _{n \rightarrow \infty} \operatorname{sp}_{\varepsilon}^{m} C_{n}(a)=\operatorname{sp}_{\varepsilon}^{m} L(a)
$$

for all $\varepsilon \in(0, \infty)$ with the exception of at most finitely many $\varepsilon_{1}, \ldots, \varepsilon_{\ell}$, where $\ell$ is not greater than the number of bounded components of $\mathbf{C} \backslash a(\mathbf{T})$. We conjecture that (44) is in fact true for all $\varepsilon \in(0, \infty)$.

We can prove (44) for all $\varepsilon \in(0, \infty)$ in the case where $a(t)=t+\alpha^{2} t^{-1}(t \in \mathbf{T})$ with $\alpha \in[0,1]$ and $m \leq 3$. For $m=3$, the proof is based on the fact that

$$
\left\|P_{3} L\left((a-\lambda)^{-1}\right) P_{3}\right\|=\sqrt{1+\frac{|\lambda|^{2}}{2}+\sqrt{|\lambda|^{2}+\frac{|\lambda|^{4}}{4}}}
$$

is nowhere locally constant in the ellipse $G_{+}$. We conjecture that for $m \geq 4$ the norms $\left\|P_{m} L\left((a-\lambda)^{-1}\right) P_{m}\right\|$ are also nowhere locally constant in $G_{+}$. 


\section{Acknowledgement}

The work of the second author was supported by UK Engineering and Physical Sciences Research Council Grant GR/M12414.

\section{References}

[1] R. M. Beam and R. F. Warming: The asymptotic spectra of banded Toeplitz and quasi-Toeplitz matrices. SIAM J. Sci. Comput. 14 (1993), 971-1006.

[2] A. Böttcher: Pseudospectra and singular values of large convolution operators. J. Integral Equations Appl. 6 (1994), 267-301.

[3] A. Böttcher, M. Embree, and V. I. Sokolov: Infinite Toeplitz and Laurent matrices with localized impurities. Linear Alg. Appl. (to appear).

[4] E. B. Davies: Spectral properties of random non-self-adjoint matrices and operators. Proc. Roy. Soc. London Ser. A 457 (2001), 191-206.

[5] U. Elsner, V. Mehrmann, F. Milde, R. A. Römer, and M. Schreiber: The Anderson model of localization: a challenge for modern eigenvalue methods. SIAM J. Sci. Comput. 20 (1999), 2089-2102.

[6] J. Feinberg and A. Zee: Non-Hermitian localization and delocalization. Phys. Rev. E 59 (1999), 6433-6443.

[7] E. Gallestey, D. Hinrichsen, and A. J. Pritchard: Spectral value sets of closed linear operators. Proc. Roy. Soc. London Ser. A 456 (2000), 1397-1418.

[8] B. Gustafson, H.-O. Kreiss, and A. Sundström: Stability theory of difference approximations for mixed initial boundary value problems, II. Math. Comput. 26 (1972), 649-686.

[9] R. Hagen, S. Roch, and B. Silbermann: $C^{*}$-Algebras in Numerical Analysis. Marcel Dekker, New York and Basel, 2001.

[10] N. Hatano and D. R. Nelson: Vortex pinning and non-Hermitian quantum mechanics. Phys. Rev. B 77 (1996), 8651-8673.

[11] F. Hausdorff: Set Theory. Chelsea, New York, 1957.

[12] D. Hinrichsen and B. Kelb: Spectral value sets: a graphical tool for robustness analysis. Systems Control Lett. 21 (1993), 127-136.

[13] D. Hinrichsen and A. J. Pritchard: Real and complex stability radii: a survey. In: D. Hinrichsen and B. Mårtensson, eds., Control of Uncertain Systems (Prog. Systems Control Theory, Vol. 6, Birkhäuser Verlag, Basel 1990), 119-162. 
[14] H.-O. Kreiss: Stability theory for difference approximations of mixed initial boundary value problems, I. Math. Comput. 22 (1968), 703-714.

[15] H. J. Landau: On Szegö's eigenvalue distribution theory and non-Hermitian kernels. J. d'Analyse Math. 28 (1975), 335-357.

[16] D. R. Nelson and N. M. Shnerb: Non-Hermitian localization and population biology. Phys. Rev. E 58 (1998), 1383-1403.

[17] L. Reichel and L. N. Trefethen: Eigenvalues and pseudo-eigenvalues of Toeplitz matrices. Linear Algebra Appl. 162-164 (1992), 153-185.

[18] R. Remmert: Funktionentheorie 1. Fourth edition, Springer-Verlag, Berlin and Heidelberg, 1995.

[19] P. Schmidt and F. Spitzer: The Toeplitz matrices of an arbitrary Laurent polynomial. Math. Scand. 8 (1960), 15-38.

[20] G. Strang, X. Liu, S. Ott, and L. He: Localized eigenvectors from localized matrix modifications (in preparation). See also G. Strang: "From the SIAM President", SIAM News, April 2000, May 2000.

[21] L. N. Trefethen, M. Contedini, and M. Embree: Spectra, pseudospectra, and localization for random bidiagonal matrices. Commun. Pure Appl. Math. (to appear).

MSC 2000: Primary 47B80

Secondary 15A18, 47B35, 60H25, 65F15, 82B44

Böttcher and Lindner

Fakultät für Mathematik

TU Chemnitz

D - 09107 Chemnitz

Germany
Embree

Oxford University Computing Laboratory

Wolfson Building, Parks Road

Oxford OX1 3QD

United Kingdom

Albrecht.Boettcher@Mathematik.TU-Chemnitz.de

Mark.Embree@comlab.ox.ac.uk

Marko.Lindner@Mathematik.TU-Chemnitz.de 